Ed. 35_Vol. 9_N. ${ }^{\circ} 3$ Septiembre_Diciembre Publicación trimestral ISSN: $2254-4143$

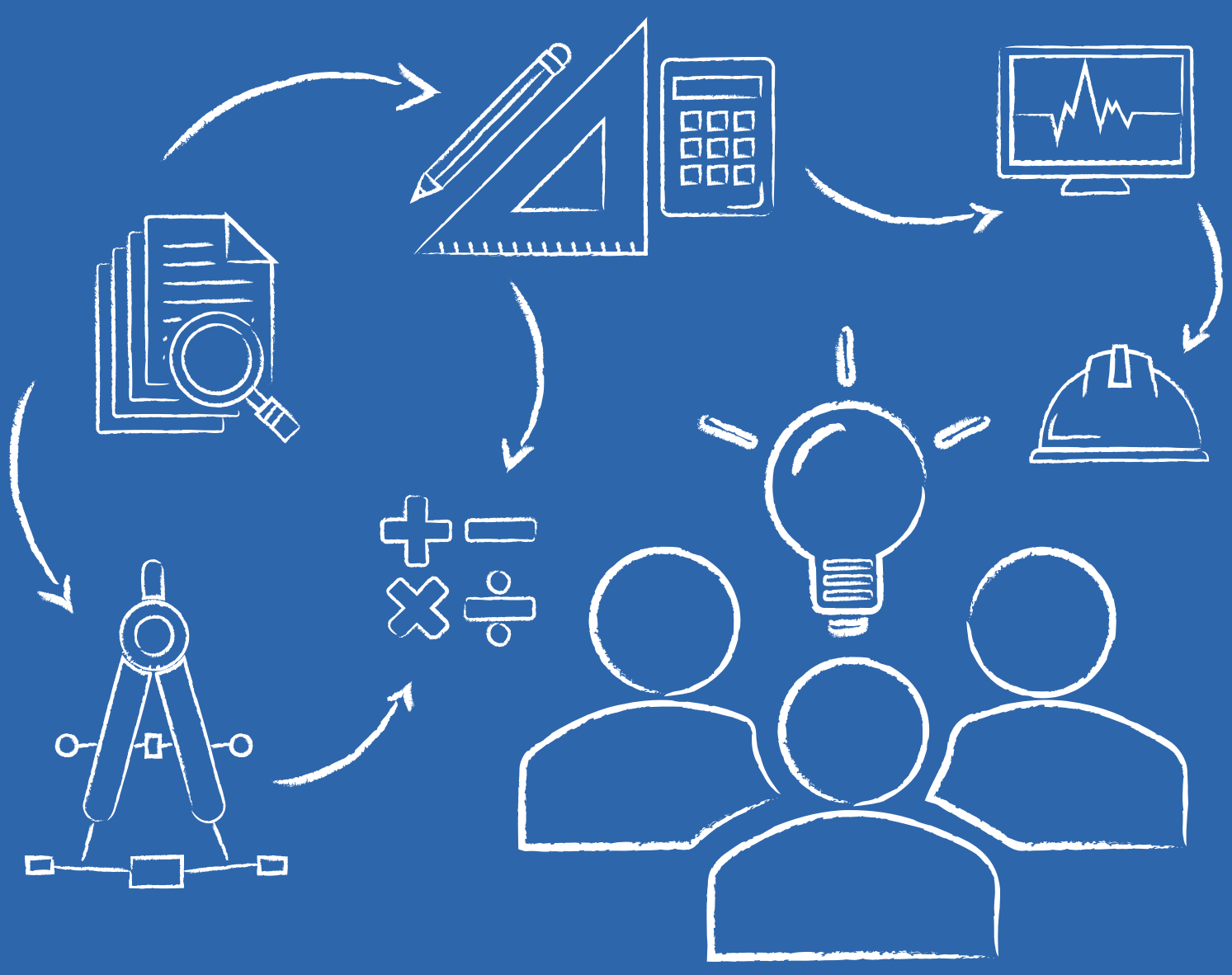




\section{G Tecnología. Glosas de innovación aplicadas a la pyme.}

Periodicidad trimestral. Quarterly periodicity.

Edición 35, Volumen 9, Número 3 (Septiembre - Diciembre).

Edition 35, Volume 9, Issue 3 (September - December).

Tirada nacional e internacional. National and internacional circulation.

Artículos revisados por el método de evaluación de pares de doble ciego.

Articles reviewed by the double blind peer evaluation method.

ISSN: $2254-4143$

Nº de Depósito Legal: A $268-2012$

DOI: https://doi.org/10.17993/3ctecno/2020.v9n3e35

Edita:

Área de Innovación y Desarrollo, S.L.

C/Alzamora 17, Alcoy, Alicante (España)

Tel: 965030572

info@3ciencias.com_www.3ciencias.com

\section{(a) $(1) \Theta$}

Todos los derechos reservados. Se autoriza la reproducción total o parcial de los artículos citando la fuente y el autor.

This publication may be reproduced by mentioning the source and the authors.

Copyright (C) Área de Innovación y Desarrollo, S.L. 


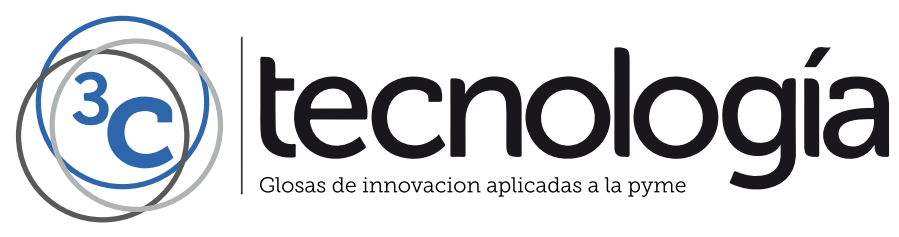




\section{CONSEJO EDITORIAL EDITORIAL BOARD}

Director

Editores adjuntos

Editores asociados
Víctor Gisbert Soler

María J. Vilaplana Aparicio

Maria Vela Garcia

David Juárez Varón

F. Javier Cárcel Carrasco

\section{CONSEJO DE REDACCIÓN DRAFTING BOARD}

Dr. David Juárez Varón. Universidad Politécnica de Valencia (España)

Dr. Martín León Santiesteban. Universidad Autónoma de Occidente (México)

Dr. F. Javier Cárcel Carrasco. Universidad Politécnica de Valencia (España)

Dr. Alberto Rodríguez Rodríguez. Universidad Estatal del Sur de Manabi (Ecuador)

\section{CONSEJO ASESOR ADVISORY BOARD}

Dra. Ana Isabel Pérez Molina. Universidad Politécnica de Valencia (España)

Dr. Julio C. Pino Tarragó. Universidad Estatal del Sur de Manabi (Ecuador)

Dr. Jorge Francisco Bernal Peralta. Universidad de Tarapacá (Chile)

Dr. Roberth O. Zambrano Santos. Instituto Tecnológico Superior de Portoviejo (Ecuador)

Dr. Sebastián Sánchez Castillo. Universidad de Valencia (España)

Dra. Sonia P. Ubillús Saltos. Instituto Tecnológico Superior de Portoviejo (Ecuador)

Dr. Jorge Alejandro Silva Rodríguez de San Miguel. Instituto Politécnico Nacional (México) 


\section{CONSEJO EDITORIAL EDITORIAL BOARD}

Área téxtil

Área financiera

Organización de empresas y RRHH

Estadística; Investigación operativa

Economía y empresariales

Sociología y Ciencias Políticas

Derecho

Ingeniería y Tecnología

Tecnologías de la Información y la Comunicación

Ciencias de la salud
Dr. Josep Valldeperas Morell

Universidad Politécnica de Cataluña (España)

Dr. Juan Ángel Lafuente Luengo

Universidad Faime I (España)

Dr. Francisco Llopis Vañó

Universidad de Alicante (España)

Dra. Elena Pérez Bernabeu

Universidad Politécnica de Valencia (España)

Dr. José Joaquín García Gómez

Universidad de Almería (España)

Dr. Rodrigo Martínez Béjar

Universidad de Murcia (España)

Dra. María del Carmen Pastor Sempere

Universidad de Alicante (España)

Dr. David Juárez Varón

Universidad Politécnica de Valencia (España)

Dr. Manuel Llorca Alcón

Universidad Politécnica de Valencia (España)

Dra. Mar Arlandis Domingo

Hospital San Fuan de Alicante (España) 


\section{POLÍTICA EDITORIAL}

\section{OBJETIVO EDITORIAL}

La Editorial científica 3Ciencias pretende transmitir a la sociedad ideas y proyectos innovadores, plasmados, o bien en artículos originales sometidos a revisión por expertos, o bien en los libros publicados con la más alta calidad científica y técnica.

\section{COBERTURA TEMÁTICA}

3C Tecnología es una revista de carácter científico-social en la que se difunden trabajos originales que abarcan la Arquitectura y los diferentes campos de la Ingeniería, como puede ser Ingeniería Mecánica, Industrial, Informática, Eléctrica, Agronómica, Naval, Física, Química, Civil, Electrónica, Forestal, Aeronáutica y de las Telecomunicaciones.

\section{NUESTRO PÚBLICO}

- Personal investigador.

- Doctorandos.

- Profesores de universidad.

- Oficinas de transferencia de resultados de investigación (OTRI).

- Empresas que desarrollan labor investigadora y quieran publicar alguno de sus estudios. 


\section{AIMS AND SCOPE}

\section{PUBLISHING GOAL}

3Ciencias wants to transmit to society innovative projects and ideas. This goal is reached thought the publication of original articles which are subdue to peer review or thorough the publication of scientific books.

\section{TEMATIC COVERAGE}

3C Tecnología is a scientific-social journal in which original works that cover Architecture and the different fields of Engineering are disseminated, such as Mechanical, Industrial, Computer, Electrical, Agronomic, Naval, Physics, Chemistry, Civil, Electronics, Forestry, Aeronautics and Telecommunications.

\section{OUR TARGET}

- Research staff.

- PhD students.

- Professors.

- Research Results Transfer Office.

- Companies that develop research and want to publish some of their works. 


\section{NORMAS DE PUBLICACIÓN}

3G Tecnología es una revista arbitrada que utiliza el sistema de revisión por pares de doble ciego (doubleblind peer review), donde expertos externos en la materia sobre la que trata un trabajo lo evalúan, siempre manteniendo el anonimato, tanto de los autores como de los revisores. La revista sigue las normas de publicación de la APA (American Psychological Association) para su indización en las principales bases de datos internacionales.

Cada número de la revista se edita en versión electrónica (e-ISSN: 2254 - 4143), identificándose cada trabajo con su respectivo código DOI (Digital Object Identifier System).

\section{PRESENTACIÓN TRABAJOS}

Los artículos se presentarán en tipo de letra Baskerville, cuerpo 11, justificados y sin tabuladores. Han de tener formato Word. La extensión será de no más de 6.000 palabras de texto, incluidas referencias. Los trabajos deben ser enviados exclusivamente por plataforma de gestión de manuscritos OJS:

https://ojs.3ciencias.com/

Toda la información, así como las plantillas a las que deben ceñirse los trabajos se encuentran en:

https://www.3ciencias.com/revista/informacion-para-autores/

https://www.3ciencias.com/normas-de-publicacion/plantillas/ 


\section{SUBMISSION GUIDELINES}

3C Tecnología is an arbitrated journal that uses the double-blind peer review system, where external experts in the field on which a paper deals evaluate it, always maintaining the anonymity of both the authors and of the reviewers. The journal follows the standards of publication of the APA (American Psychological Association) for indexing in the main international databases.

Each issue of the journal is published in electronic version (e-ISSN: $2254-4143$ ), each work being identified with its respective DOI (Digital Object Identifier System) code.

\section{PRESENTATION WORK}

The papers will be presented in Baskerville typeface, body 11 , justified and without tabs. They must have Word format. The extension will be no more than 6.000 words of text, including references. Papers must be submitted exclusively by OJS manuscript management platform:

https://ojs.3ciencias.com/

All the information, as well as the templates to which the works must adhere, can be found at:

https://www.3ciencias.com/en/journals/infromation-for-authors/

https://www.3ciencias.com/en/regulations/templates/ 


\section{ESTRUCTURA}

Los trabajos originales tenderán a respetar la siguiente estructura: introducción, métodos, resultados, discusión/conclusiones, notas, agradecimientos y referencias bibliográficas.

Es obligatoria la inclusión de referencias, mientras que notas y agradecimientos son opcionales. Se

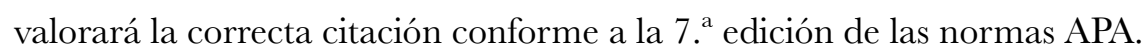

\section{RESPONSABILIDADES ÉTICAS}

No se acepta material previamente publicado (deben ser trabajos inéditos). En la lista de autores firmantes deben figurar única y exclusivamente aquellas personas que hayan contribuido intelectualmente (autoría), con un máximo de 4 autores por trabajo. No se aceptan artículos que no cumplan estrictamente las normas.

\section{INFORMACIÓN ESTADÍSTICA SOBRE TASAS DE ACEPTACIÓN E INTERNACIONALIZACIÓN}

- $\quad$ Número de trabajos aceptados publicados: 5.

- Nivel de aceptación de manuscritos en este número: 38,5\%.

- $\quad$ Nivel de rechazo de manuscritos: 61,5\%.

- Internacionalización de autores: 4 países (Pakistán, India, Perú y México).

Normas de publicación: https://www.3ciencias.com/revista/informacion-para-autores/ 


\section{STRUCTURE}

The original works will tend to respect the following structure: introduction, methods, results, discussion/ conclusions, notes, acknowledgments and bibliographical references.

The inclusion of references is mandatory, while notes and acknowledgments are optional. The correct citation will be assessed according to the 7 th edition of the APA standards.

\section{ETHICAL RESPONSIBILITIES}

Previously published material is not accepted (they must be unpublished works). The list of signatory authors should include only and exclusively those who have contributed intellectually (authorship), with a maximum of 4 authors per work. Articles that do not strictly comply with the standards are not accepted.

\section{STATISTICAL INFORMATION ON ACCEPTANCE AND INTERNATIONALIZATION FEES}

- Number of accepted papers published: 5 .

- Level of acceptance of manuscripts in this number: 38,5\%.

- Level of rejection of manuscripts: $61,5 \%$.

- Internationalization of authors: 4 countries (Pakistan, India, Peru and Mexico).

Guidelines for authors: https://www.3ciencias.com/en/journals/infromation-for-authors/ 


\section{INDEXACIONES INDEXATIONS}

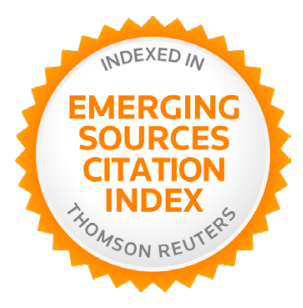

latindex
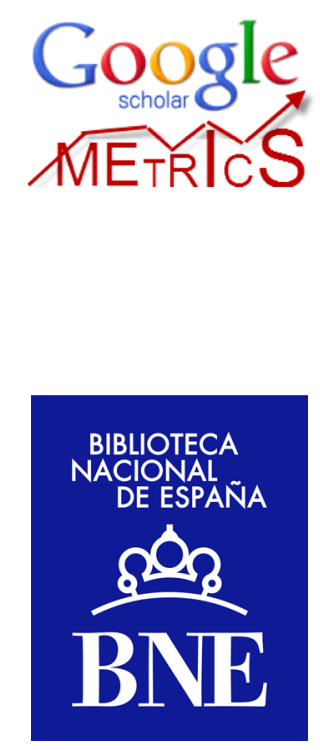

EBSCOhost
ERIHPLUK
MIAR per a l'Avaluació de Revistes
DULCINEA

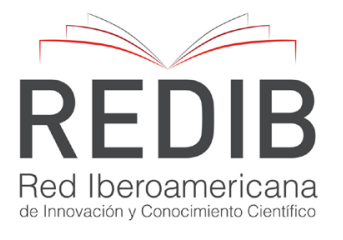

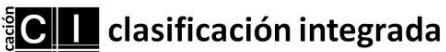

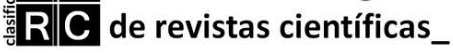

\section{OAJ Open Academic .net}

o SHERPA/RøMEO 


\section{INDEXACIONES INDEXATIONS}

\section{- Dialnet}
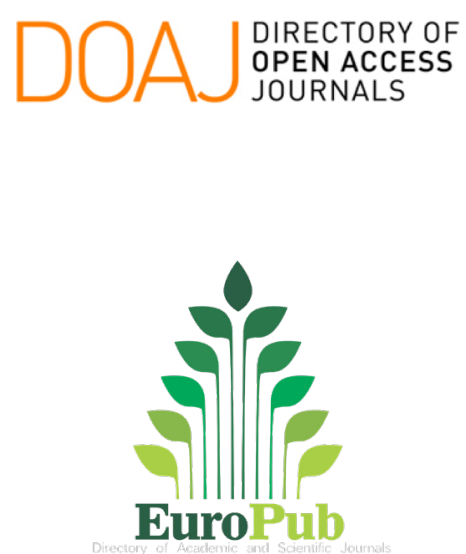

CSIC

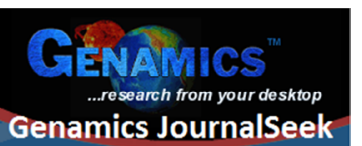

Genamics JournalSeek

\section{ÍnDICES}

ST WorldCat

\section{Crossref}




\section{/SUMARIO/ /SUMMARY/}


Sistema de inteligencia de negocios para soporte de decisiones en la comercialización de plantas ornamentales

\section{Business intelligence system for decision making in the commercialization of ornamental plants}

Héctor Adán Morales Lugo, Patricia Elizabeth Figueroa Millán, Nicandro Farías Mendoza y Ramona Evelia Chávez Valdez

Mix design with response surface methodology to optimize the flexural strength of concrete

A novel method for improving Bit Error Rate in sensor networks by using Orthogonal Space Time Block Code (OSTBC) coding

Lemna minor influence in the treatment of organic pollution of the industrial effluents

Alternate formula for calculating the Darcy Coefficient in turbulent flow in pipes 


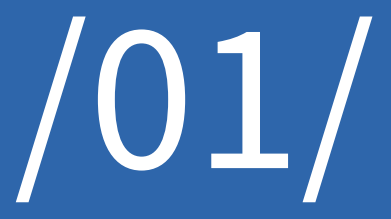




\section{SISTEMA DE INTELIGENCIA DE NEGOCIOS PARA SOPORTE DE DECISIONES EN LA COMERCIALIZACIÓN DE PLANTAS ORNAMENTALES

BUSINESS INTELLIGENCE SYSTEM FOR DECISION MAKING IN
THE COMMERCIALIZATION OF ORNAMENTAL PLANTS

Héctor Adán Morales Lugo

Estudiante de Maestría del Tecnológico Nacional de México, Instituto Tecnológico Nacional de México/I.T. Colima,

(México).

E-mail: rotceh1296@hotmail.com ORCID: https://orcid.org/0000-0002-1407-3361

Patricia Elizabeth Figueroa Millán

Profesor adjunto de la División de Estudios de Posgrado del Tecnológico Nacional de México/I.T. Colima, (México).

E-mail: patricia.figueroa@colima.tecnm.mx ORCID: https://orcid.org/0000-0001-7562-7578

Nicandro Farías Mendoza

Profesor adjunto de la División de Estudios de Posgrado del Tecnológico Nacional de México/I.T. Colima, (México). E-mail:nfarias@colima.tecnm.mx ORCID: https://orcid.org/0000-0001-5543-0719

Ramona Evelia Chávez Valdez

Profesor adjunto de la División de Estudios de Posgrado del Tecnológico Nacional de México/I.T. Colima, (México). E-mail: echavez@colima.tecnm.mx ORCID: https://orcid.org/0000-0002-5697-6825

Citación sugerida:

Morales, H.A., Figueroa, P.E., Farias, N., y Chávez, R.E. (2020). Sistema de inteligencia de negocios para soporte de decisiones en la comercialización de plantas ornamentales. 3C Tecnología. Glosas de innovación aplicadas a la pyme, 9(3), 17-45. https://doi.org/10.17993/3ctecno/2020.v9n3e35.17-45 


\section{RESUMEN}

La horticultura ornamental en México es una industria en crecimiento que requiere la inclusión de diversas tecnologías para automatizar la producción y comercialización a fin de incrementar su rentabilidad. Para esto, el análisis de los datos es clave, permitiendo la obtención de conocimiento para el soporte a la toma de decisiones; no obstante, implica un tiempo exhaustivo de procesamiento de información, afectando la productividad de las empresas debido a la falta de un sistema de apoyo a la toma de decisiones que implemente herramientas dinámicas de inteligencia de negocios. Este trabajo de investigación propone un sistema web de inteligencia de negocios para la creación de herramientas dinámicas y ejecución de consultas asíncronas a la base de datos; lo cual, proporciona un análisis de la información histórica de la comercialización de plantas ornamentales mediante tablas, gráficas y reportes. Está desarrollado utilizando la metodología PUA, el lenguaje de programación Python y el framework Django, empleando un enfoque innovador al aplicar el algoritmo DFS como mecanismo de búsqueda para determinar la relación existente entre las tablas de la base de datos, reduciendo tiempo de extracción, procesamiento, análisis y presentación de información. Como resultado se logró mejorar el aprovechamiento de la información histórica, eficientar el procesamiento y análisis de la información de comercialización y, por consiguiente, mejorar los procesos de toma de decisiones.

\section{PALABRAS CLAVE}

Algoritmo DFS, Horticultura ornamental, Sistema de inteligencia de negocios, DSS, Consultas SQL dinámicas. 


\section{ABSTRACT}

Ornamental horticulture in Mexico is a growing industry that requires the inclusion of various technologies to automate production and marketing in order to increase its profitability. For this, data analysis is key, allowing obtaining knowledge to support decision-making; However, it involves exhaustive information processing time, affecting the productivity of companies due to the lack of a decision support system that implements dynamic business intelligence tools. This research work proposes a business intelligence web system for the creation of dynamic tools and execution of asynchronous queries to the database; which provides an analysis of the historical information on the commercialization of ornamental plants through tables, graphs and reports. It is developed using the PUA methodology, the Python programming language and the Django framework, employing an innovative approach by applying the DFS algorithm as a search mechanism to determine the relationship between the database tables, reducing extraction time, processing, analysis and presentation of information. As a result, it was possible to improve the use of historical information, streamline the processing and analysis of marketing information and, consequently, improve decision-making processes.

\section{KEYWORDS}

Ornamental horticulture, Business intelligence system, DFS algorithm, DSS, Dynamic SQL queries. 


\section{INTRODUCCIÓN}

La horticultura es un segmento de la agricultura relacionado no solo con la cultura vegetal, conocida como horticultura alimentaria; sino también, con un gran número de especies como plantas para el embellecimiento de espacios físicos, a lo cual se le conoce como horticultura ornamental. La horticultura involucra diferentes áreas altamente relacionadas que ofrecen grandes oportunidades para educación, recursos, industrias profesionales, tecnología y empleo (Paiva, 2018). En cuanto a lo anterior, la tecnología ha demostrado ser una herramienta clave para mejorar la capacidad productiva, la competitividad y la comercialización de productos, incluyendo los productos de la horticultura ornamental.

Sin embargo, para que la horticultura ornamental en México pueda ser considerada como una industria ornamental con alcances internacionales de competitividad, se requiere de grandes pasos para solventar los problemas de hardware, conocimiento, infraestructura y cooperación, ya que se observa la inclusión de la tecnología en tres niveles de estratificación: bajo, medio y alto; lo cual, afecta negativamente cuestiones como el análisis, priorización, toma de decisiones y diseño de futuros sistemas (García, van der Valk, y Elings, 2011). Además, la focalización es un factor clave para asegurar el valor implícito de la información, como elemento principal para la toma de decisiones; es decir, es fundamental que se determine el conjunto de variedades estratégicas de las flores y plantas de ornato con la finalidad de orientar la generación de bases mínimas de información y así comprender el funcionamiento de la oferta y las necesidades específicas de la demanda (Coepplants Colima A. C., 2012).

Por consiguiente, el análisis de datos históricos es un factor clave para generar estrategias de comercialización y producción, permitiendo la obtención de conocimiento para el soporte a la toma de decisiones. No obstante, para las habilidades de un humano es un proceso complejo, tedioso, ineficaz e ineficiente; lo cual, afecta la productividad de las empresas. Ante esto, surgen distintas estrategias para utilizar los datos de las empresas y convertirlos en información útil para la toma de decisiones, tal es el caso de las herramientas de inteligencia de negocios (BI, por sus siglas en inglés) y los sistemas de apoyo a la toma de decisiones (DSS, por sus siglas en inglés). Las herramientas BI combinan la 
obtención y almacenamiento de datos con herramientas analíticas que presentan información compleja y competitiva a los tomadores de decisiones (Roldán, Cepeda-Carrión, y Galán, 2012). Se utilizan en la generación, tratamiento y comunicación de la información, proporcionando una visión estratégica del negocio para transformar grandes cantidades de datos en información de calidad; asimismo, proveen recursos como análisis, pronóstico, monitoreo, control y optimización para gestionar la calidad de los procesos y tomar decisiones oportunas (Silva, Silva y Gomes, 2016). Por otro lado, los sistemas DSS, son considerados como sistemas de información basados en ordenadores que combinan modelos y datos, tienen la finalidad de resolver problemas semiestructurados con una amplia implicación del usuario, permitiendo otorgar herramientas que posibilitan la toma de decisiones analítica y cuantitativa con base en la información resultante (Power, 2011).

Ambos términos son muy similares, ya que permiten "tomar decisiones comerciales"; además, se enfocan en apuntalar el proceso de toma de decisiones en las empresas de manera eficiente y eficaz con base en los datos de éstas. A pesar de esto, los sistemas BI no siguen una estructura fija como los DSS, es decir, su estructura no es estable, permitiendo a los desarrolladores de soluciones de inteligencia de negocios cubrir solo algunos de los componentes de un DSS o ampliar su funcionalidad según el contexto al que será aplicado (Kopackova y Škrobáčková, 2006).

Existen distintos casos de estudios, hasta donde se tiene conocimiento, que describen el desarrollo e implementación de diversos sistemas BI para apoyar a la toma de decisiones, no necesariamente aplicados al sector hortícola ornamental, pero que consideran puntos claves en la importancia de la implementación de este tipo de sistemas en las empresas. En particular, Medeiros (2017), describe el desarrollo e implementación de un sistema BI para apoyar a la toma de decisiones en los procesos de gestión académica, el cual está desarrollado en Excel, implementando un cubo de datos y un gran número de herramientas para procesar y visualizar la información de forma dinámica; sin embargo, no es accesible desde cualquier dispositivo, ya que las tecnologías empleadas se utilizan en un ámbito local, imposibilitando el acceso, procesamiento y análisis remoto de la información. 
Damasceno, Azevedo, y Pinto (2018) describen la implementación de un sistema BI mediante un Data Mart, un Data Warehouse y un Cubo de Datos, utilizando la herramienta Pentaho. Éste permite visualizar la información de una base de datos dinámicamente mediante tablas y gráficas; sin embargo, se desarrolló como una aplicación para escritorio que no puede ser utilizada por dispositivos remotos y que además, requiere de conocimiento de las herramientas, el lenguaje SQL y bases de datos para su configuración y que además, no muestra un resultado preciso sobre el potencial de la herramienta, los beneficios o su eficiencia.

Castro et al. (2019) plantean la implementación de un tablero o dashboard BI aplicado a la gestión administrativa; en el cual, visualizan por medio de un tablero o dashboard la información mediante gráficas a partir de la información solicitada por formularios de Google y de la extracción de información de una BD; sin embargo, se describe como una solución para un procesamiento básico de la información.

En el caso de estudio de Santillán y Mendoza (2015), se desarrolló un sistema web de apoyo a ejecutivos que, mediante la generación de gráficas y reportes, apoya a la toma eficiente de decisiones en los procesos de compras. La información es extraída de una base datos histórica; sin embargo, no otorga al usuario herramientas de inteligencia de negocios que le permitan realizar un análisis y una toma de decisiones eficiente; además, no permite manipular la información dinámicamente.

Por otro lado, Zimmermann (2006) describe un sistema BI para escritorio que ayuda a tomar decisiones a partir de reportes utilizando un componente de Delphi y un cubo de datos para generar la información extraída de la base de datos. Permite la visualización de los datos mediante tablas, gráficas y reportes; sin embargo, las tecnologías utilizadas son para la actualidad obsoletas y requiere de conocimiento básico de SQL para realizar un cambio de base de datos, consultas, etc. No utiliza ningún tipo de algoritmo para automatizar el proceso de consultas dinámicas.

Como se puede observar, en la literatura existen pocos ejemplos de sistemas BI para la horticultura ornamental, aun cuando la mayoría de los casos de estudio descritos anteriormente presentan una buena 
alternativa para apoyar a la toma de decisiones; sin embargo, estos cuentan con ciertas limitaciones y áreas de oportunidad que pueden aprovecharse ya que son:

- Desarrollados o implementados como aplicaciones de escritorio; por lo cual, para las demandas de la actualidad, se puede considerar como una ventaja y área de oportunidad, el desarrollo de una alternativa web para el acceso remoto al sistema y a la información analizada por éste.

- Implementados con tecnologías obsoletas, interfaces o dashboards no muy amigables para el usuario; por lo tanto, la utilización de tecnologías de vanguardia y de acceso a abierto posibilita el acceso ubicuo al sistema BI.

- Diseñados para ser manejados por un experto, sin contar con una alternativa para los usuarios no expertos en el tema.

Así mismo, la empresa ORNACOL, dedicada a la comercialización de plantas ornamentales en la ciudad de Colima, cuenta con un sistema web que permite administrar los procesos de comercialización de plantas ornamentales y seguir la trazabilidad hacia adelante, en donde los datos históricos son almacenados en una base de datos y son utilizados para el funcionamiento del sistema (García-Mejía, García-Virgen, y Chávez-Valdez, 2018) modificando los bienes y servicios que se intercambian, así como los modos en que se producen, almacenan, distribuyen, transportan y comercializan. Este nuevo escenario abre una variedad de interrogantes para las políticas comerciales. La trazabilidad surge como consecuencia de cambios en los hábitos de los consumidores, quienes exigen cada vez más seguridad alimentaria (recolección, elaboración, almacenaje, distribución, etc.. Este sistema genera grandes cantidades de información sobre la comercialización de plantas ornamentales, que está siendo desaprovechadas, ya que el sistema no cuenta con un sistema de apoyo a la toma de decisiones que le permita automatizar los procesos manuales de procesamiento y análisis de la información que la empresa lleva a cabo. Utilizando correctamente la información histórica se puede disminuir los tiempos de procesamiento, análisis y apoyar al proceso de toma de decisiones de la empresa sobre la comercialización de las plantas 
ornamentales, con la finalidad de comprender el funcionamiento de la oferta y las necesidades específicas de la demanda como se mencionó anteriormente.

Por lo tanto, tomando como caso de estudio la empresa ORNACOL, en este artículo se el desarrollo y aplicación de un sistema BI para el soporte en la toma de decisiones sobre la comercialización de plantas ornamentales. Éste permite resolver problemas semiestructurados, tomando los datos históricos de comercialización generados por el sistema descrito anteriormente, con la finalidad de proporcionar sugerencias o soluciones complejas dirigidas a estos datos. Este sistema BI es desarrollado por fases e implementado como un sistema web basado en lenguajes de programación de uso libre como Python y Django para el procesamiento y análisis eficiente de datos. Utiliza el estilo arquitectural REST para la construcción de WEB APIs y la ejecución de peticiones asíncronas al sistema de comercialización previamente mencionado. Este sistema permite aprovechar la información generada por el sistema de comercialización y apoyar en la toma de decisiones para la generación de mejores estrategias de comercialización.

Así pues, las principales características del sistema BI propuesto permiten cumplir los siguientes objetivos:

- Consultar información a la base de datos histórica.

- Creación de herramientas de inteligencia de negocios con interfaces ergonómicas, que permiten la visualización y manipulación de información en tiempo cuasi-real.

- Reducción de tiempo de procesamiento y análisis de cantidades masivas de información.

- Mejorar la toma de decisiones.

- Comprender el funcionamiento de la oferta y las necesidades específicas de la demanda.

Por lo tanto, en este trabajo se describe un nuevo enfoque para la aplicación de sistemas BI en el sector de la horticultura ornamental, contribuyendo a solucionar unas de las problemáticas encontradas de manera específica en el estado de Colima, como se establece en el Plan Estratégico para la Operación 
Anual del Componente de Desarrollo de Capacidades y Extensionismo Rural del Estado de Colima, siendo éstas: el cultivo de plantas sin una planeación en el mercado y el uso de canales de comercialización inadecuados.

Este sistema, como un mecanismo de soporte en el proceso de toma de decisiones, permitirá en un futuro la planificación de las especies a cultivar orientadas al mercado mediante la identificación de las variedades requeridas o demandadas por éste y, por consiguiente, generar mejores estrategias de comercialización. Cabe señalar, que este sistema puede ser extrapolado o contextualizado a cualquier otro sistema producto.

\section{METODOLOGÍA}

El desarrollo de la investigación se llevó a cabo en las instalaciones de la empresa ORNACOL en el municipio de Coquimatlán, Colima, fundamentándose en una investigación mixta, utilizando el enfoque cualitativo para hacer un estudio sobre el impacto de la implementación del sistema en la empresa, y cuantitativo para precisar la misma y dar solución a las problemáticas del sector en cuanto a la comercialización de plantas ornamentales.

La arquitectura del sistema propuesto sigue la arquitectura de un DSS con las características de contextualización de un sistema BI y, debido a la utilización de una metodología por fases para el desarrollo del software, se diseñó de la siguiente manera:

- Descripción de los tipos de problemas semiestructurados: punto principal de un sistema de inteligencia de negocios con soporte para la toma de decisiones, ya que éstos requieren del conocimiento humano para lograr las decisiones finales.

- Especificación de la taxonomía de interacción activa: proporciona sugerencias o soluciones estables a problemas complejos. 
- Especificación de la taxonomía de uso dirigida a datos: enfatiza la recuperación de datos de una base de datos histórica.

- Metodología: en este caso se decidió utilizar una metodología por fases llamada Proceso Unificado Ágil (AUP, por sus siglas en inglés) para el desarrollo del software, la cual se describe a continuación.

Jacobson, Brooch, y Rumbaugh (1999), establecen cuatro fases y siete disciplinas en las cuales se lleva a cabo el desarrollo del sistema iterativo incremental (véase Figura 1). Debido a que la propuesta de diseño del sistema requiere una metodología por fases, se optó por desarrollar el sistema web BI siguiendo esta metodología ágil.

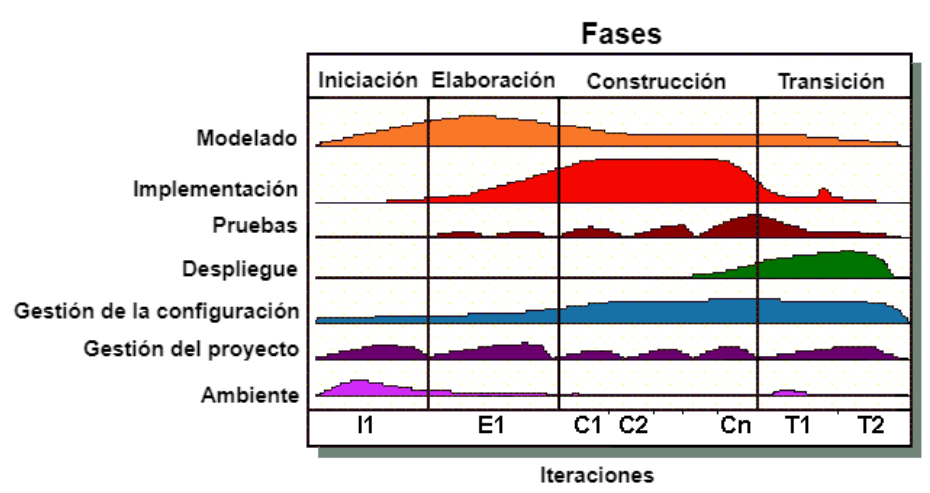

Figura 1. Metodología Proceso unificado Ágil (AUP). Fuente: adaptado de (Ambler, 2014).

Como se puede observar en la Figura 1, en cada disciplina se desarrolla una actividad diferente que permite mitigar los riesgos de implementación del software y asegurar un desarrollo óptimo.

La arquitectura lógica del sistema, considera la arquitectura lógica de un DSS, la cual consta de cuatro componentes fundamentales: una base de conocimientos o base de datos, un modelo matemático, una interfaz de usuario y los propios usuarios (Marakas, 1999). Por otro lado, la arquitectura física del sistema se fundamenta en una arquitectura cliente-servidor, como se muestra en la Figura 2. 


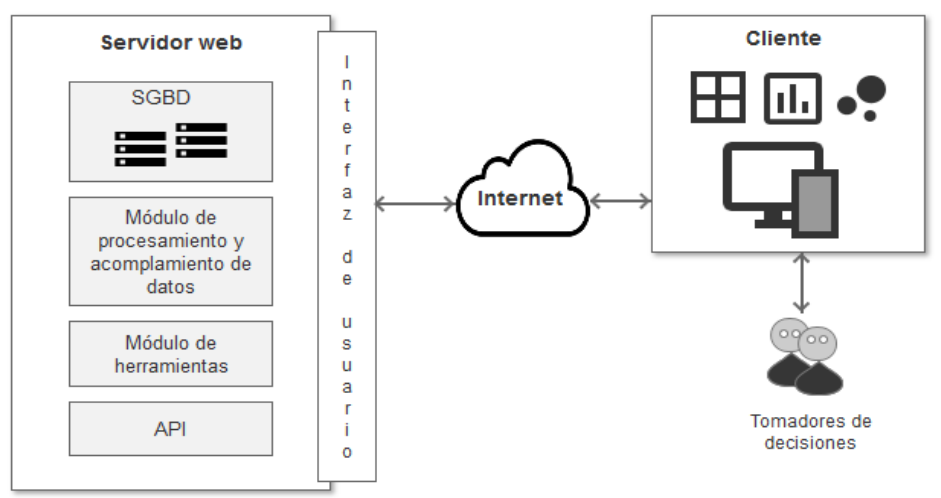

Figura 2. Arquitectura cliente-servidor de ORNALISIS. Fuente: elaboración propia.

Como se puede observar en la Figura 2, la arquitectura especifica dos módulos: una interfaz de aplicaciones (API, por sus siglas en ingles) y un sistema gestor de base de datos (SGDB), un módulo de procesamiento y acoplamiento de datos, y el módulo de herramientas dinámicas. La funcionalidad de estos módulos se describe a continuación:

- SGBD: contiene la información histórica de la comercialización de plantas ornamentales; además, se especificó un base de datos propia para el sistema BI.

- API: permite consultar información de manera asíncrona a la base de datos desde cualquier dispositivo utilizando el formato JSON para solicitar y desplegar información.

- Módulo de procesamiento y acoplamiento de datos: se encarga de acoplar y procesar los datos recibidos y enviados a través de la interfaz de aplicaciones (API, por sus siglas en ingles), la información es convertida a formato JSON y regresada al cliente para su posterior despliegue en forma de tabla, gráficas y reportes.

- Módulo de herramientas: el módulo de herramientas incluye las herramientas dinámicas, y herramientas integradas: 
- Las herramientas dinámicas extraen información de la base de datos histórica mediante parámetros, permitiendo la creación de consultas dinámicas a la base de datos con la implementación del algoritmo DFS para la obtención de relaciones entre tablas. Este algoritmo DFS se implementó debido que el sistema permite vincular cualquier BD relacional, ya que el sistema está dirigido a usuarios no expertos en la materia; por lo tanto, se necesitaba un método para la construcción de consultas dinámicas que permita convertir cualquier BD relacional a grafo y después determinar mediante DFS si se puede ejecutar la consulta o no, sin requerir un conocimiento sobre la estructura de la base de datos por el usuario del sistema.

El sistema construye dinámicamente una consulta SQL ejecutable por MySQL, llevando a cabo los siguientes pasos:

1. Obtener la información que el usuario solicita en el cliente o navegador web.

2. Procesar la información y solicitar la información interna de la BD seleccionada.

3. Construir las cláusulas SELECT y WHERE a partir de la información solicitada por el usuario.

4. Construir la cláusula FROM, que contiene las tablas utilizadas en la consulta y aplicar el algoritmo DFS diseñado para determinar la existencia de relaciones entre las tablas.

5. Juntar todas las cláusulas y ejecutar la consulta SQL.

- Las herramientas integradas, consultan información a la base de datos de comercialización y permiten visualizar información específica mediante tablas, gráficas y reportes personalizados tal cual y como la empresa los requiera. Estas herramientas no cuentan con la función de una dinámica ya que están dirigidas a consultas muy específicas, 
es decir, son herramientas que en este caso en particular la empresa ORNACOL requiere mensualmente para la extracción de información. Por ejemplo, ventas por especie, ventas totales por año, entre otras.

\section{RESULTADOS}

Se implementó un sistema web BI de apoyo a la toma de decisiones para la comercialización de plantas ornamentales mediante el framework web Django, el cual posibilita un procesamiento rápido de la información y el escalamiento futuro del sistema. Además, se utilizó el framework Django REST para la construcción de la Web API que establece un acceso seguro a los datos a través de políticas de autenticación, así como la ejecución de consultas asíncronas para incrementar el rendimiento de éste, ya que permite atender nuevas solicitudes mientras se encuentra procesando una solicitud previa.

Por lo tanto, el sistema web propuesto y descrito en este artículo, denominado ORNALISIS, permite la creación de herramientas dinámicas para realizar un análisis específico de la información de la comercialización de plantas ornamentales (véase Figura 3), dando la posibilidad al operador de manipular parámetros, tablas y gráficas para realizar consultas asíncronas a la base de datos y extraer la información requerida para la toma de decisiones, sin necesidad de ser un experto en el área. Cada una de las herramientas proporcionadas despliega: el tipo de usuario, el usuario que creó la herramienta, un icono que indica si el usuario es propietario de la herramienta, un icono que indica si es una herramienta compartida con otro usuario y un icono de opciones para editar o borrar la herramienta. 


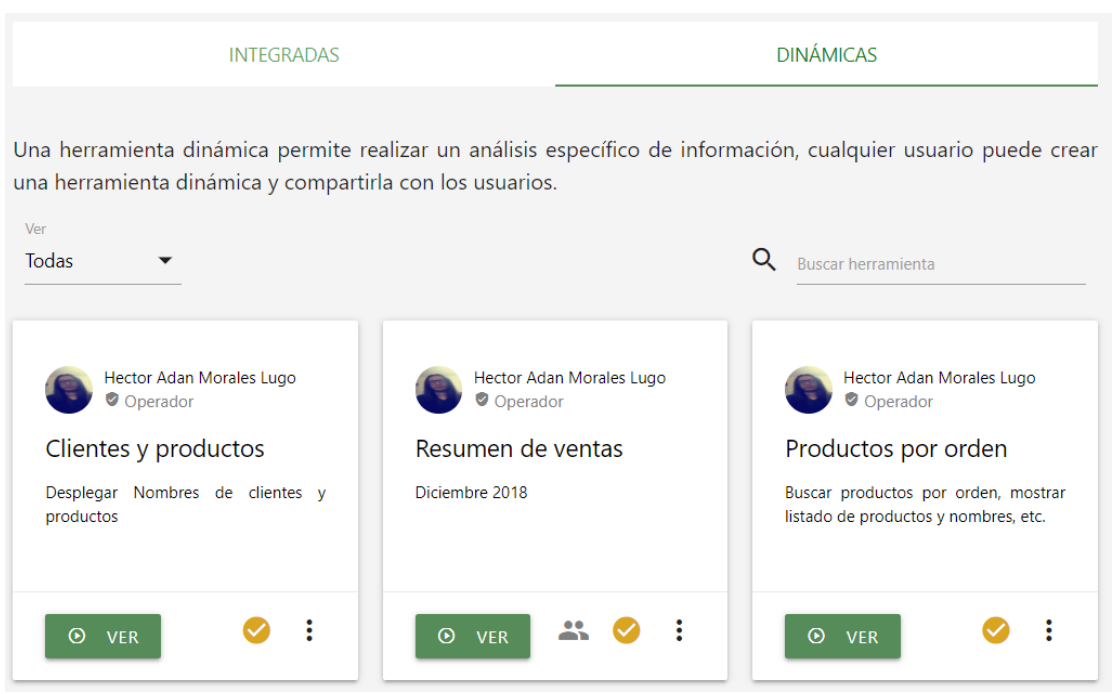

Figura 3. Panel de herramientas dinámicas. Fuente: elaboración propia.

Por otro lado, en la Figura 4 se muestra un modal para la selección de las tablas necesarias para el análisis de la información, esta opción permite al usuario seleccionar la base de datos y tablas con los cuales trabajar. El sistema determina si es posible utilizar dichos parámetros para el análisis de la información de manera interna y transparente para el usuario. Este sistema está diseñado para trabajar con bases de datos relacionales, ya que éstas se han considerado las más consistentes y seguras, así como las más utilizadas principalmente por las empresas, posibilitando con esto la contextualización y extrapolación del sistema propuesto a otros sistemas productos. 


\section{Selecciona las tablas a utilizar.}

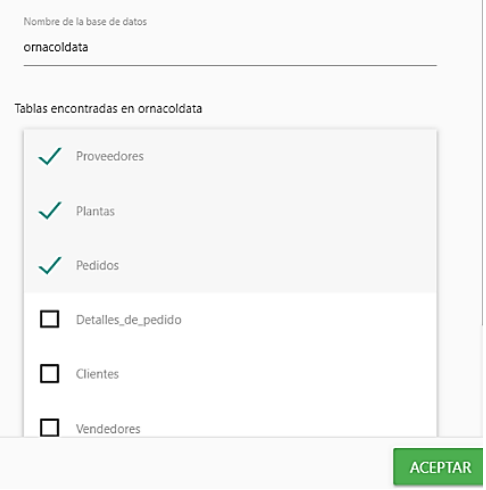

Figura 4. Configuración inicial de una herramienta dinámica. Fuente: elaboración propia.

Posteriormente, el usuario debe configurar su herramienta a través del panel de elementos (véase Figura 5) que permite agregar parámetros, tablas y gráficas a la herramienta.

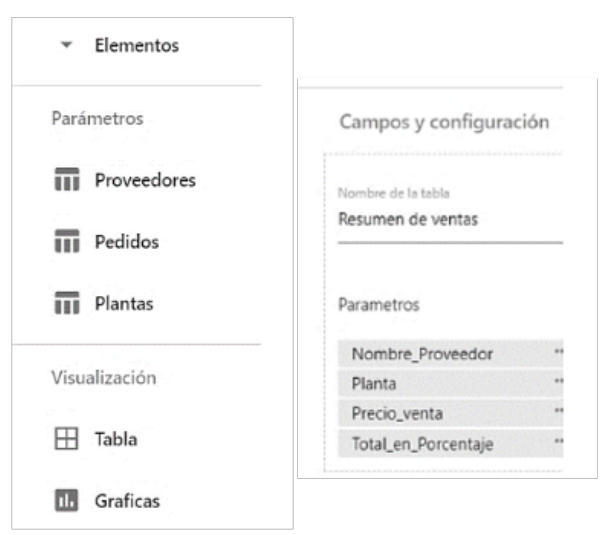

Figura 5. Panel de elementos de una herramienta dinámica. Fuente: elaboración propia.

El panel de herramientas permite configurar la tabla y gráfica a visualizar (véase Figura 6), con la finalidad de facilitar la consulta de información y otorgar al usuario un control total de la información y representación visual de ésta. 


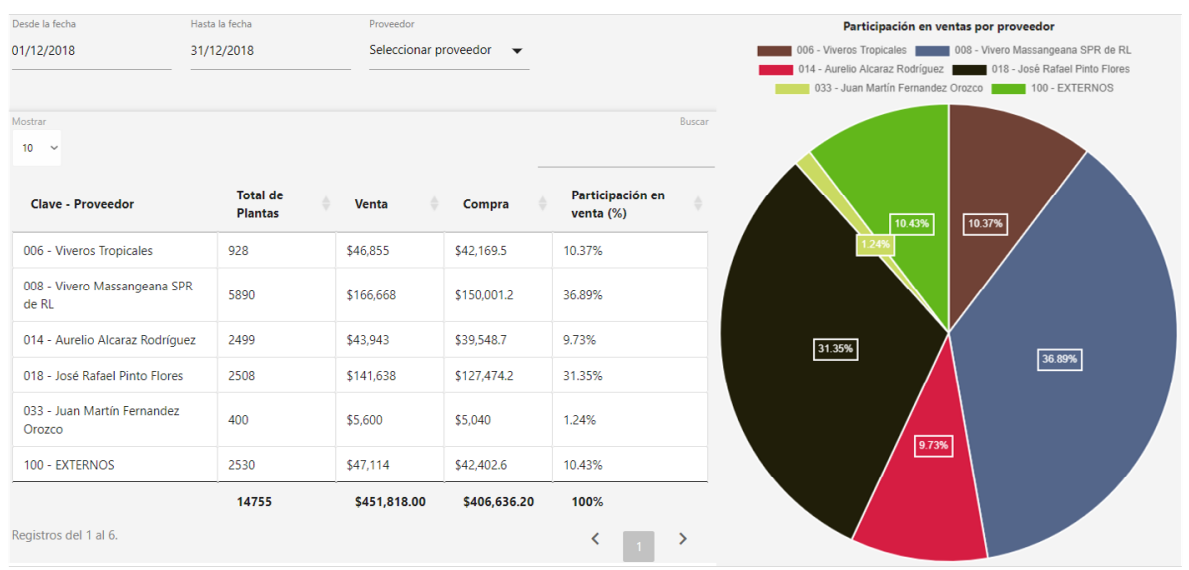

Figura 6. Herramienta dinámica con parámetros, tabla y gráfica. Fuente: elaboración propia.

\subsection{CONSTRUCCIÓN DE CONSULTAS DINÁMICAS}

El sistema se encarga de procesar las peticiones que le son enviadas a través de la Web API, un conjunto de direcciones URL que se encargan de recibir y enviar datos en formato JSON. Dependiendo de los elementos añadidos en la herramienta, se sigue un proceso que involucra algoritmos que construyen dinámicamente una consulta SQL entendible por el gestor de base de datos MySQL. Este proceso se describe en el diagrama de flujo de la Figura 7. 

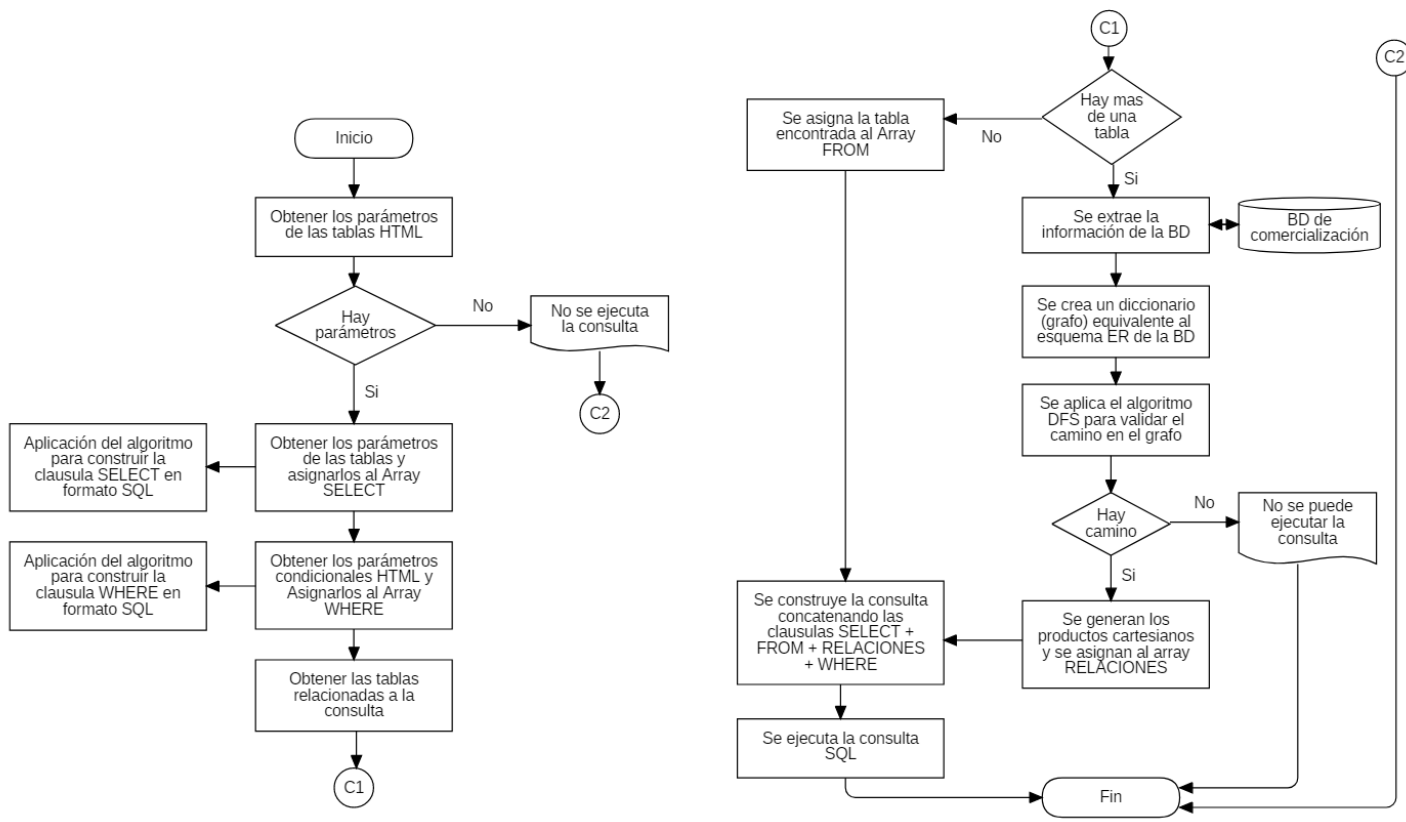

Figura 7. Diagrama de flujo para la construcción de consultas dinámicas. Fuente: elaboración propia.

Como se puede observar en la Figura 7, la creación del grafo y utilización del algoritmo DFS se usarán siempre y cuando se hayan encontrado más de una tabla, esto debido a que se puede utilizar cualquier BD y de la cual se desconocen las relaciones entre las tablas. A continuación, se muestra la explicación a detalle de dicho proceso, el cual se realiza empatando los elementos HTML de la interfaz de usuario, como tablas y gráficas que se desean visualizar, con sus respectivos parámetros a instrucciones SQL:

- Las tablas por visualizar en el navegador web se traducen a una instrucción SELECT de SQL, determinando con esto las columnas que se seleccionarán de cierta tabla de la base de datos mediante los parámetros seleccionados. Cada parámetro contiene información de la tabla a la que pertenece, el nombre de la columna y sus valores. Si se añaden tres parámetros (columnas) a la tabla, la variable SELECT almacenará el nombre de las tres columnas, asignándose a una variable de tipo array de la siguiente manera: 
SELECT = "nombre_proveedor, planta, precio_venta"

- Las gráficas toman los parámetros de la tabla construida en el navegador web y despliegan el resultado. Las gráficas aceptan parámetros y funcionan igual que las tablas; por lo tanto, al modificar un valor, se realiza una consulta que filtra la información solicitada y despliega los resultados en la gráfica.

- Los parámetros se traducen a la instrucción WHERE de SQL, la cual determina los filtros para la información, si el usuario modifica la información, ésta se actualizará y generará una nueva consulta. Si se añade por ejemplo el parámetro fecha y se selecciona un valor para éste, la variable WHERE almacenará la columna y el valor seleccionado, especificándose como se establece a continuación:

WHERE = “fecha BETWEEN '2018-01-01' AND '2018-12-01'”

Los parámetros, como ya se mencionó anteriormente, pueden ser de varios tipos y estos equivalen a elementos y controles HTML (select, select multiple, input para texto, input para fecha, input para checkbox, etc.).

A partir de los parámetros y tablas, el sistema crea la instrucción FROM a través de la utilización del array FROM, definiéndose como se muestra a continuación:

FROM = "proveedores, productos, ordenes"

Éste permite determinar cuáles tablas se utilizarán para realizar la consulta a partir de una búsqueda de la información de la base de datos que deduce cuáles tablas están relacionadas entre sí para determinar si es posible realizar la consulta, mediante la implementación del algoritmo DFS. Además, si el usuario realiza la petición de una consulta compleja (más de dos tablas), el sistema se encargará de realizar un JOIN o producto cartesiano añadiéndolo al WHERE para relacionar todas las tablas seleccionadas, y realizar la consulta de forma correcta. 


\subsection{ALGORITMO PARA LA CREACIÓN DE GRAFOS}

Para determinar las relaciones que corresponden a la consulta y construir los productos cartesianos, se aplican una serie de algoritmos que ayudan a resolver el problema. Primeramente, el algoritmo de creación de grafos genera un diccionario en Python que equivale al esquema ER de la base de datos, el cual determina las relaciones de cada tabla en la misma para formar el grafo, donde se crea una consulta a la base de datos donde se obtiene el nombre de la tabla, la llave primaria o columna que relaciona a esa tabla con otra, el tipo de relación, y la tabla relacionada a la misma.

SELEGT table_name, column_name, constraint_name, referenced_table_name, referenced_column_ name FROM information_schema.key_column_usage WHERE table_schema = db

Con esta información se genera un grafo con los nombres en string de cada tabla y las tablas relacionadas a la misma (véase Figura 8).

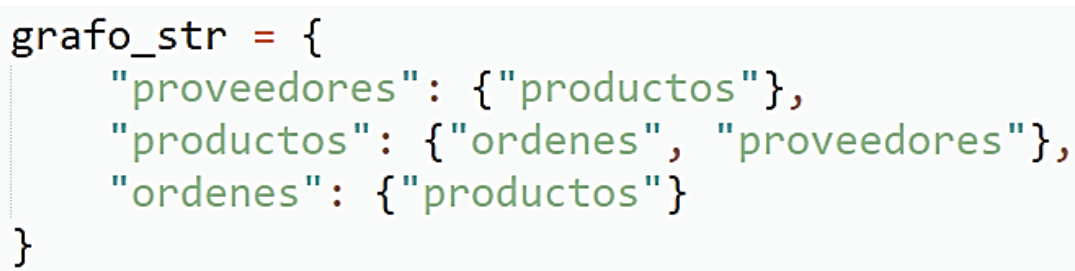

Figura 8. Representación de un grafo en diccionario de Python. Fuente: elaboración propia.

Después, a cada tabla se le asigna un número y se crea el grafo con equivalentes numéricos (véase Figura 9).

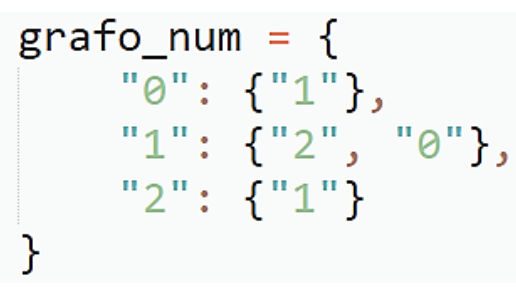

Figura 9. Representación de un grafo en diccionario de Python con etiquetas numéricas. Fuente: elaboración propia. 
El grafo numérico representado con un diccionario, grafo_num en la Figura 9, se toma como parámetro por el algoritmo DFS. Este grafo contiene la información de las relaciones y las tablas, determinando si esas tablas están relacionadas entre sí, generando como resultado una serie de caminos que se analizaron para definir las relaciones que se utilizarán en la consulta.

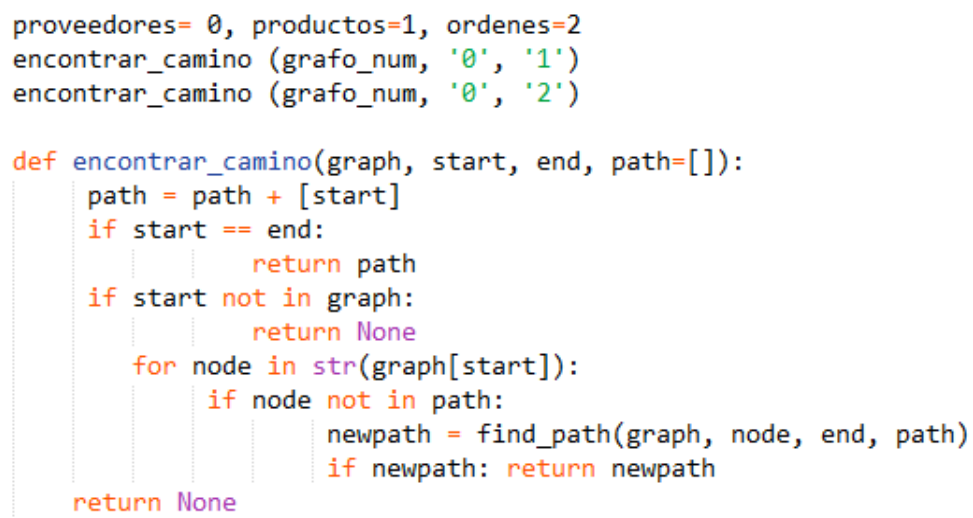

Figura 10. Algoritmo DFS que busca un camino entre dos puntos. Fuente: elaboración propia.

A partir de los caminos encontrados por el algoritmo de búsqueda en profundidad DFS, se generan los productos cartesianos con las relaciones encontradas y se asignan a la variable RELACIONES.

Relaciones $=$ "proveedires.producto_id == productos.producto_id AND productos.orden_id == oredenes.orden_id"

Al final, las cuatro variables (SELECT, FROM, WHERE, RELACIONES) se concatenan y forman la consulta final a ejecutarse (véase Figura 11).

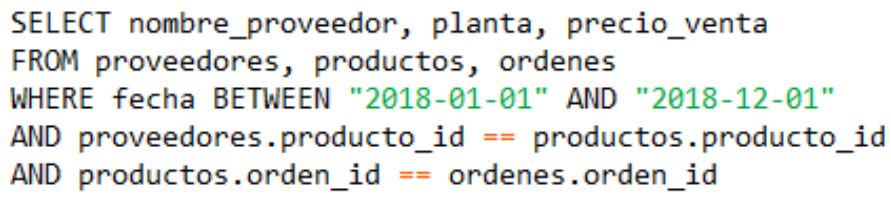

Figura 11. Consulta SQL construida dinámicamente. Fuente: elaboración propia. 
Para este caso, el sistema solicita información de la base de datos histórica externa al sistema BI y perteneciente al sistema de comercialización de ORNACOL para después regresar el resultado de todo el proceso descrito anteriormente, de manera totalmente transparente para el usuario utilizando el plugin jQuery DataTables, como se observa en la Figura 6.

Por otra parte, el sistema permite generar reportes y exportarlos en formato XLSX o PDF para ser presentados formalmente (véase la Figura 12), dando la posibilidad al usuario de seleccionar la información necesaria a guardar y la configuración del documento.

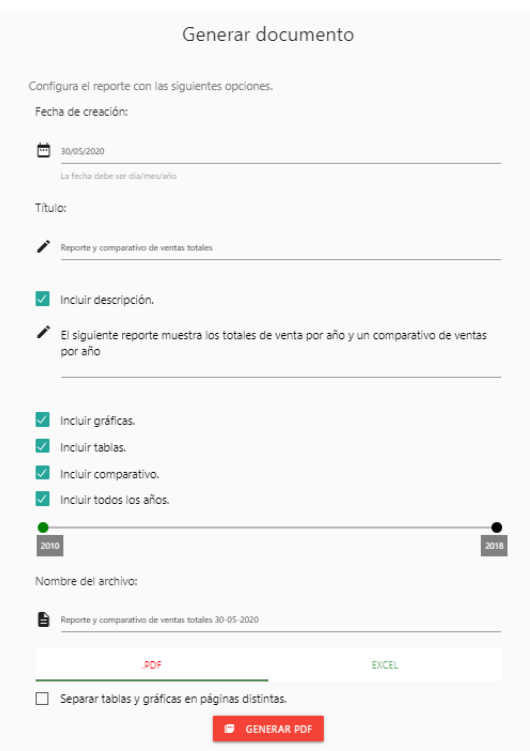

Figura 12. Generación de reportes en formato PDF y xlsx. Fuente: elaboración propia.

El algoritmo para procesar y agrupar la información se codificó específicamente para realizar consultas a bases de datos relacionales, este mismo es capaz de recibir datos por el método POST utilizando AJAX, procesarlas para formar la consulta final a ejecutarse en la base de datos y regresar un resultado al usuario para realizar un análisis de la información y tomar decisiones finales. 
Por otro lado, el diseño de la Web API utilizando Django REST, permite al sistema tener un mejor rendimiento y no interrumpir las interacciones del usuario con el sistema; no obstante, es importante analizar el tiempo que tarda en realizar una consulta compleja (más de dos tablas) donde resulte una gran cantidad de datos, ya que el sistema responderá dependiendo el tipo de conexión y equipo que se tenga (véase Tabla 1).

Tabla 1. Tiempos de respuesta de consultas realizadas por el usuario al servidor con equipos con distintas características.

\begin{tabular}{|c|c|c|c|}
\hline Equipo & Conexión & Tipo de consulta & Tiempo promedio \\
\hline \multirow{2}{*}{$\begin{array}{l}\text { Computadora de } \\
\text { escritorio, procesador Intel } \\
\text { Core i7 7700K, 8GB RAM }\end{array}$} & \multirow{2}{*}{$\begin{array}{l}\text { Por cable, } \\
10 \mathrm{MB} \text { de } \\
\text { velocidad }\end{array}$} & $\begin{array}{c}\text { Simple ( } 2 \text { tabla) } \\
2 \mathrm{~K} \text { datos }\end{array}$ & $30 \mathrm{~ms}$ \\
\hline & & $\begin{array}{c}\text { Compleja (más de dos } \\
\text { tablas) } \\
100 \mathrm{~K} \text { datos }\end{array}$ & $100 \mathrm{~ms}$ \\
\hline \multirow{2}{*}{$\begin{array}{l}\text { Laptop, procesador Intel } \\
\text { Celeron, 4GB RAM }\end{array}$} & \multirow[b]{2}{*}{ Wifi, $10 \mathrm{MB}$} & $\begin{array}{l}\text { Simple (2 tabla) } \\
2 \mathrm{~K} \text { datos }\end{array}$ & $250 \mathrm{~ms}-450 \mathrm{~ms}$ \\
\hline & & $\begin{array}{c}\text { Compleja (más de dos } \\
\text { tablas) } \\
100 \mathrm{~K} \text { datos }\end{array}$ & $250 \mathrm{~ms}-1.2 \mathrm{~s}$ \\
\hline \multirow{2}{*}{$\begin{array}{l}\text { Computadora de } \\
\text { escritorio, Intel Core i7 } \\
860,4 \text { GB de RAM }\end{array}$} & \multirow{2}{*}{$\begin{array}{l}\text { Por cable, } \\
10 \mathrm{MB} \text { de } \\
\text { velocidad }\end{array}$} & $\begin{array}{c}\text { Simple (1 tabla) } \\
2 \mathrm{~K} \text { datos }\end{array}$ & $250 \mathrm{~ms}-450 \mathrm{~ms}$ \\
\hline & & $\begin{array}{c}\text { Compleja (más de dos } \\
\text { tablas) } \\
100 \mathrm{~K} \text { datos }\end{array}$ & $250 \mathrm{~ms}-800 \mathrm{~ms}$ \\
\hline
\end{tabular}

Fuente: elaboración propia.

Como se puede observar en la Tabla 1, los tiempos de respuesta con diferentes equipos se miden desde que el usuario genera una consulta al servidor, se procesa, se ejecutan los algoritmos de generación de grafos búsqueda en profundidad, construcción de consultas, extracción de información de la consulta generada y hasta el regreso y presentación de un resultado al cliente. 
Por otro lado, en cuanto a la evaluación del impacto del sistema se realizó una comparativa entre el tiempo de procesamiento de los datos con la implementación del sistema y el tiempo sin la implementación de éste. Por lo cual, es importante señalar que las actividades de análisis de información en la empresa para la generación de reportes semanales, mensuales y anuales se realizaban de manera manual, estimándose un promedio de 1 a 3 horas para realizar este procesamiento de información. En contraste, con la implementación del sistema este tiempo de procesamiento se redujo significativamente, como se puede observar en la Figura 13.

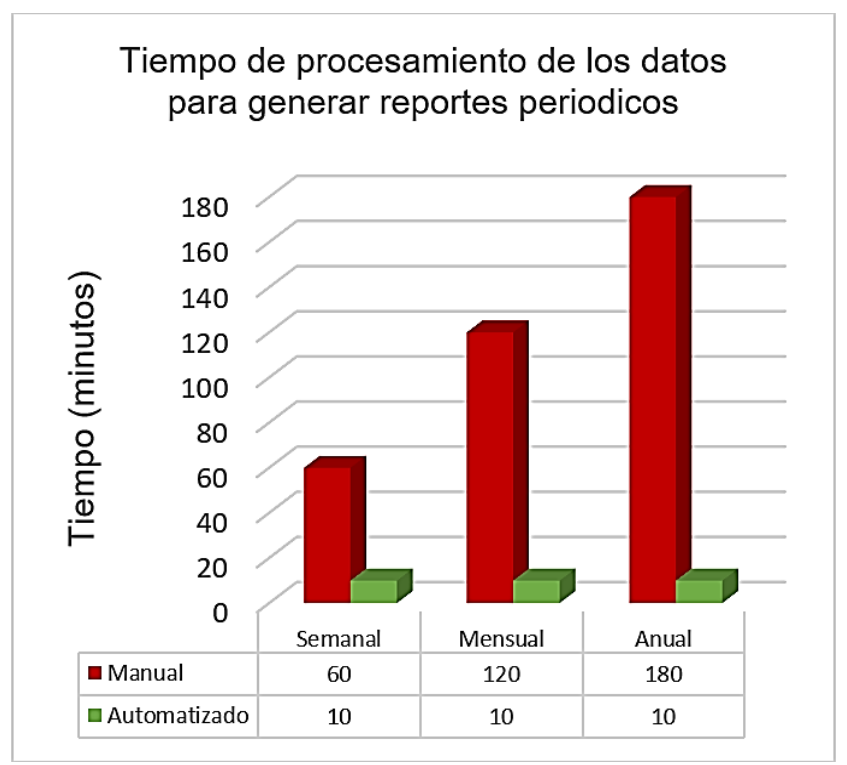

Figura 13. Tiempo de procesamiento de los datos para generar reportes periódicos (manual= persona, automatizado= sistema). Fuente: elaboración propia.

Antes de la implementación del sistema, el proceso de análisis de información se realizaba de forma manual en hojas de cálculo, lo cual implicaba la inversión de varias horas de trabajo por parte del operador para extraer de un software de contabilidad toda la información necesaria de las ventas y guardarla en una hoja de cálculo en blanco para después limpiarla, agruparla y asociarla para generar el reporte de ventas y con base en ello tomar decisiones, como se puede observar en la Figura 13. Proceso 
que se realizaba de manera semanal, mensual y anual, siendo un proceso ineficiente e ineficaz, sesgando la posibilidad de tomar decisiones oportunas en la comercialización de plantas ornamentales.

Considerando lo anterior, queda demostrado que la implementación del sistema BI propuesto para el apoyo en la toma de decisiones en la empresa ORNACOL, permite en primer lugar automatizar el proceso de extracción, procesamiento y presentación de la información sobre la comercialización de plantas de ornato. Contribuyendo con lo anterior, a adquirir información de utilidad para la extracción de conocimiento que permita generar mejores y más eficientes estrategias de comercialización.

\section{CONCLUSIONES}

En este artículo se presenta un sistema web que da soporte a la toma de decisiones, automatizando y haciendo eficiente el proceso manual de recolección y agrupación de la información. El sistema permite crear herramientas dinámicas que, con base en los parámetros solicitados, construye consultas dinámicamente entendibles por gestores de bases de datos relacionales utilizando SQL; posteriormente, los datos obtenidos se procesan, serializan y se representan en el cliente por medio de tablas, gráficas y reportes. Debido a que el sistema puede establecer conexión a cualquier BD relacional, un algoritmo crea un grafo equivalente al entidad-relación de la BDR y el algoritmo DFS, a partir del grafo generado, permitiendo optimizar la búsqueda de las relaciones entre dos o más tablas para crear las consultas dinámicas.

Este tipo de herramientas podrían ayudar a desarrollar sistemas BI para aquellas empresas que busquen gestionar su información y requieran un sistema capaz de conectar cualquier base de datos relacional realizar consultas dinámicas y que pueda operarse sin necesidad de conocimientos avanzados. Considerando, además, que las bases de datos relacionales son las bases de datos mayormente utilizadas para el desarrollo de sistemas de información, proporcionando interoperabilidad con los sistemas ya existentes. 
Así pues, el sistema BI web ORNALISIS contribuye a incrementar la cantidad, calidad, rentabilidad y sustentabilidad de la producción y comercialización de plantas ornamentales, apoyando a la toma de decisiones, reduciendo el tiempo de procesamiento y análisis de grandes cantidades de información e incrementando la productividad de la empresa. Fundamentado así, el desarrollo de este trabajo de investigación.

Como trabajo futuro se espera optimizar el funcionamiento de las herramientas dinámicas y mitigar los riesgos de requerir un cierto nivel de conocimiento en bases de datos relacionales para interpretar las tablas seleccionadas, utilizar un método de búsqueda distinto para determinar el óptimo y un método distinto a uniones cruzadas para combinar filas de tablas relacionadas por ejemplo la cláusula JOIN. Finalmente se espera que el DSS pueda dar soluciones autónomas aplicando una taxonomía de uso dirigido a modelos para apoyar a los tomadores de decisiones a tomar una decisión final.

\section{AGRADECIMIENTOS}

Al Tecnológico Nacional de México campus Instituto Tecnológico de Colima y al Consejo Nacional de Ciencia y Tecnología (CONACYT).

\section{REFERENCIAS BIBLIOGRÁFICAS}

Ambler, S. W. (2014). The Agile Unified Process. The Agile Unified Process (AUP). http://www.ambysoft. $\mathrm{com} / \mathrm{scottAmbler.html}$

Castro, A. G., Fonseca, R. M., Melo, A., y Prado, A. (2019). Business Intelligence Aplicado a Gestão Acadêmica no Setor Administrativo. Revista De Tecnologia Da Informação E Comunicação Da Faculdade Estácio Do Pará, 2(3), 14. http://revistasfap.com/ojs3/index.php/tic/article/view/275 
Coepplants Colima A. G. (2012). Plan Rector: Comité Sistema Producto Ornamentales Colima. http://dev. pue.itesm.mx/sagarpa/estatales/EPT\%20COMITE\%20SISTEMA\%20PRODUCTO\%20 ORNAMENTALES\%20COLIMA/PLAN\%20REGTOR\%20QUE\%20CONTIENE\%20 PROGRAMA\%20DE\%20TRABAJO\%202012/PR_ORNAMENTALES_COLIMA_2012. pdf

\section{Comisión Estatal del Componente de Desarrollo de Capacidades y Extensionismo Rural.} (2013). Plan Estratégico para la Operación Anual del Componente de Desarrollo de Capacidades y Extensionismo Rural del Estado de Colima. Comisión Estatal del Componente de Desarrollo de Capacidades y Extensionismo Rural. http://www.inca.gob.mx/webfiles/planes-estrat-e/plan_estrategico_col. pdf

Damasceno, E., Azevedo, A., y Pinto, A. (2018). Business Intelligence-Implantation on Federal Institute of Triângulo Mineiro (IFTM) System: Proceedings of the 10th International Conference on Computer Supported Education, 528-535. https://doi.org/10.5220/0006818805280535

García, N., van der Valk, O. M. G., y Elings, A. (2011). Mexican protected horticulture: Production and market of Mexican protected horticulture described and analysed. Wageningen UR Greenhouse Horticulture/LEI (Rapporten GTB 1126). https://edepot.wur.nl/196070

García-Mejía, E. E., García-Virgen, J., y Ghávez-Valdez, R. E. (2018). Gestión de la Comercialización de Plantas Ornamentales Utilizando Normas de Trazabilidad hacia delante. RIIIT. Revista internacional de investigación e innovación tecnológica, 6(35). http://www.scielo.org.mx/ scielo.php?pid=S2007-97532018000500006\&script=sci_abstract

Jacobson, I., Brooch, G., y Rumbaugh, J. (1999). Unified Software Development Process (2a ed). AddisonWesley Professional. 
Kopackova, H., y Škrobáčková, M. (2006). Decision support systems or business intelligence: what can help in decision making? https://www.researchgate.net/publication/44982242_Decision_support_systems_ or_business_intelligence_what_can_help_in_decision_making\#: : text $=U \operatorname{sing} \%$ 20a\% 20 programmed $\% 20$ decisions $\% 20$ require,effective $\% 20$ business $\% 20$ decisions $\% 20 \% 5$ B $4 \% 5$ D $\% 20$.

Marakas, G. M. (1999). G. M. Marakas. Decision support systems in the twenty-first century (2a ed.). Prentice Hall.

Medeiros, J. D. L. (2017). Desenvolvimento de um Sistema de Inteligência de Negócios para apoio da Gestão Acadêmica. Universidade Federal De Itajubá. http://repositorio.unifei.edu.br/xmlui/ handle/123456789/1032

Paiva, P. (2018). Horticulture and Ornamental Horticulture. Ornamental Horticulture, 24(1), 6-6. https:// www.researchgate.net/publication/327666995_Horticulture_and_Ornamental_Horticulture

Power, D. J. (2011). Decision support systems: Concepts and resources for managers. Greenwood/Quorum Books.

Roldán, J. L., Gepeda-Garrión, G., y Galán, J. L. (2012). Los sistemas de inteligencia de negocio como soporte a los procesos de toma de decisiones en las organizaciones. Papeles de Economía Española, 132, 239-260. https://hdl.handle.net/11441/76099

Santillán, Á. G., y Mendoza, M. L. (2015). Diseño y desarrollo de un sistema Web de apoyo a ejecutivos para la toma eficiente de decisiones de compras. Revista Iberoamericana de Producción Académica y Gestión Educativa. https://www.pag.org.mx/index.php/PAG/article/view/284/330

Silva, R. A., Silva, F. G. A., y Gomes, G. F. S. (2016). O Uso Do Business Intelligence (Bi) Em Sistema De Apoio À Tomada De Decisão Estratégica. Revista Gestão Inovação e Tecnologias, 6(1), 2780-2798. http://www.revistageintec.net/index.php/revista/article/view/726 
Zimmermann, T. R. (2006). Desenvolvimento De Um Sistema De Apoio À Decisão Baseado Em Business Intelligence, 77 . 


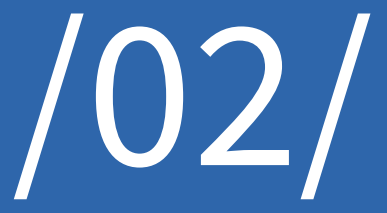




\title{
MIX DESIGN WITH RESPONSE SURFACE METHODOLOGY TO OPTIMIZE THE FLEXURAL STRENGTH OF CONCRETE
}

\author{
Freddy Lizardo Kaseng Solis \\ National University Federico Villarreal, Lima, (Perú). \\ E-mail: fkaseng@unfv.edu.pe ORCID: https:// orcid.org/0000-0002-2878-9053 \\ Luis Jimmy Clemente Gondori \\ National University Federico Villarreal, Lima, (Perú). \\ E-mail: kelvin0296@yahoo.es ORCID: https://orcid.org/0000-0002-0250-4363
}

Giro Rodriguez Rodriguez

National University Mayor de San Marcos, Lima, (Perú).

E-mail: crodriguezro@unmsm.edu.pe ORCID: https://orcid.org/0000-0003-2112-1349

Recepción: 14/05/2020 Aceptación: 08/07/2020 Publicación: 14/09/2020

\section{Citación sugerida:}

Kaseng, F.L., Clemente, L.J., y Rodriguez, C. (2020). Mix design with response surface methodology to optimize the flexural strength of concrete. 3C Tecnología. Glosas de innovación aplicadas a la pyme, 9(3), 47-57. https://doi. org/10.17993/3ctecno/2020.v9n3e35.47-57 


\section{ABSTRACT}

This paper presents a technical proposal for the application of experimental designs in the construction processes of civil works, specifically in the manufacture of concrete for rigid pavements. Currently is still does not apply these technological advances, or any application of research methods that allows optimizing processes to more satisfactory levels, despite the advantages and benefits they provide in terms of achieving unexpected performance and significant savings of component materials. The experimental design model is a classic statistical model whose objective is to determine if the independent factors influence in a variable of interest in this case the modulus of breakage of the concrete or in another important factor, during the bending test. The answer surface methodology is based on the experimentation in three stages, the results achieved allow locating an optimal area where the adjusted values lead to reduced consumption of them and expanding the strength of the concrete.

\section{KEYWORDS}

Concrete, Experimental method, Optimization, Resistance, Flexural strength, Rigid pavements. 


\section{INTRODUCTION}

The rigid pavements made based on concrete, present in their vast majority the presence of structural and superficial fissures, these have become a problem regarding its functionality and the useful life in terms of the level of service to the vehicles that circulate in these routes.

The reasons are many; among the most relevant we can name the mix design for concrete, the method of making the "cloths", the materials used, the quantity or proportion of the components, etc., the latter is constitutes the objective of the investigation. It is observed that the method used at the present time are the conventional ones that do not allow the necessary adjustments to be made to the materials and their performance in order to achieve optimum levels in terms of dosage and as a product, greater resistance to bending called the Break Module (MR).

The manufacturing method is frequently that of the American Concrete Institute (ACI), as the dosage is very rigid and closed, it does not allow modifications to be made to the components, such as cement, aggregate, water, additives, etc. For this, it is necessary to resort to other methods that give the possibility of manipulating these independent variables and with the hypothesis of reaching optimum levels of the mechanical properties of the concrete. For this it is necessary to use the experimental designs (widely used in other areas of engineering) that, based on a practical methodology and the use of logical reasoning, it is possible to adjust both the quantity of materials and the resistance to flexion even more optimal, is possible use algortihms for calculations as Sánchez et al. (2020) and Soto et al. (2020).

Deterministic simulation models have the characteristic of being a technique for solving practical problems (Levy et al., 2020), such as an overall change of variables over time. Experimental models are an approximation to the real system. From the above, there is a need to repeat multiple simulation runs, consequently, its use in an investigation should be planned as a series of experiments that lead to significant interpretations of the relationships of interest (Huapaya, Rodriguez \& Esenarro, 2020). 
The response surface methodology (MSR) was used in this investigation. This modeling methodology is a holistic approach that allows us to postulate the form of the objective function, update and limit the values of the parameters, as well as explore and approach the region close to the optimal estimate.

\section{METHOD}

The Response Surface Methodology (MSR) is the tool used to achieve the proposed objectives, for which reason it was necessary to experiment sequentially in the stages it comprises until the desired level of improvement is found. In this case, after a first experimental stage (selection of influential variables) it was necessary to move the experimental region (move from place) in a suitable direction, or to explore the initial experimental region in more detail (see Figure 1). The way to do both is part of the so-called Response Surface Methodology (MSR).

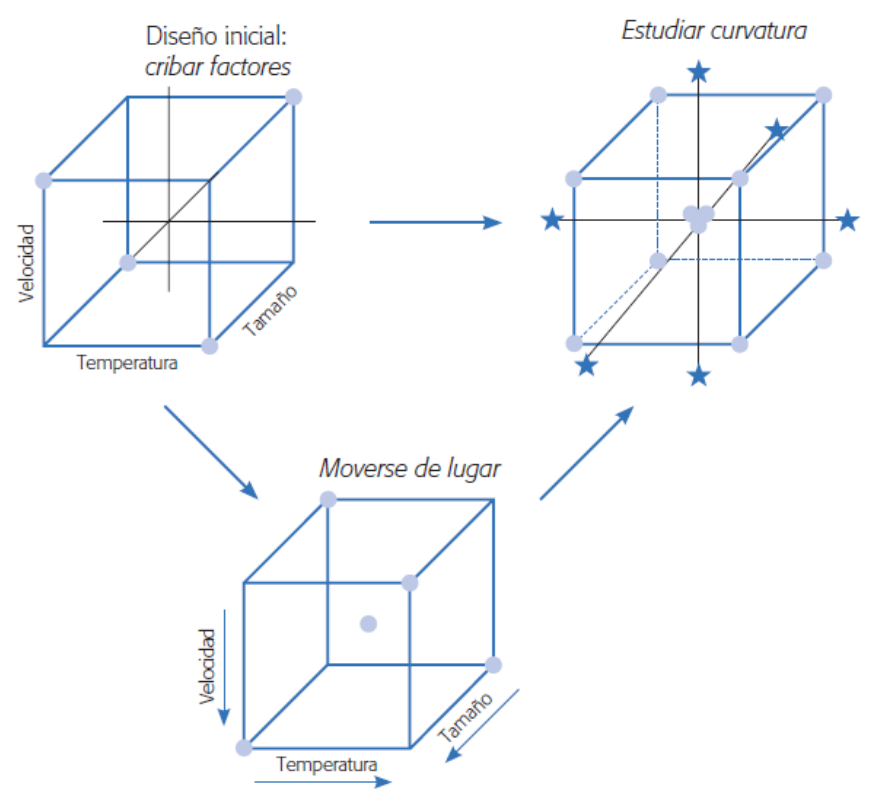

Figure 1. Basic actions of the MSR. Source: (Gutiérrez-Pulido \& De la Vara, 2008). 


\subsection{SIEVING OR CLASSIFICATION}

In this first optimization stage, we identify the controllable variables that can significantly influence the process responses (fine aggregate, cement) and "eliminate" those that were not significant for a good economic orientation of the process, because it reduces the number of variables and Experimental tests in the later stages of optimization (Figure 2). When controllable variables range from low to high, they affect expected responses. For our case, we use the first-order factorial design.

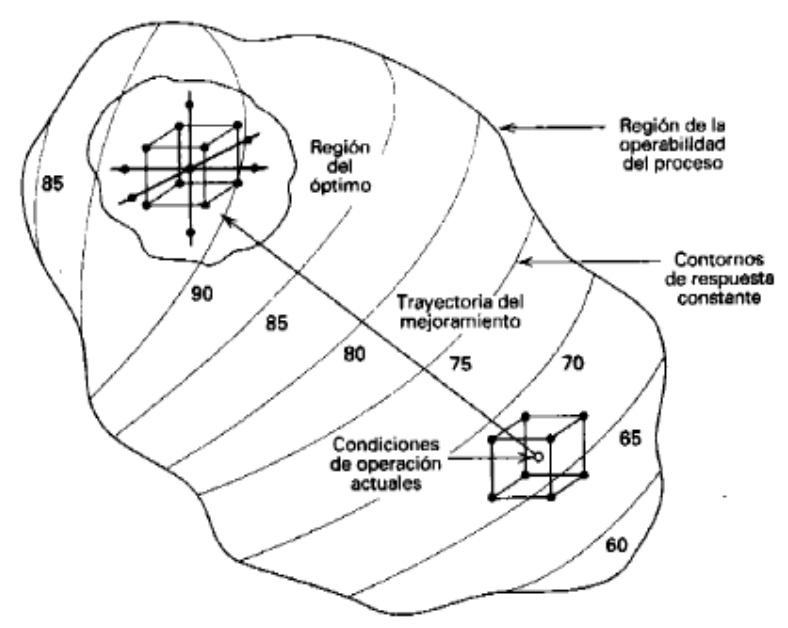

Figure 2. The sequence of the MSR. Source: (Montgomery \& Runger, 1996).

\subsection{ESCALATION}

Having located the optimal region, and observing that it is still far from the initial experiments (sieving), then was initiated the second stage called "scaling", which consisted of successively climbing towards the optimal region until reaching it (Figure 3), to achieve for this objective, we use the indirect method of ascending slopes in two periods. The method of the steepest ascent is the one that allowed us to go to the zone of the maximum increase of the response, according to formula (1). 


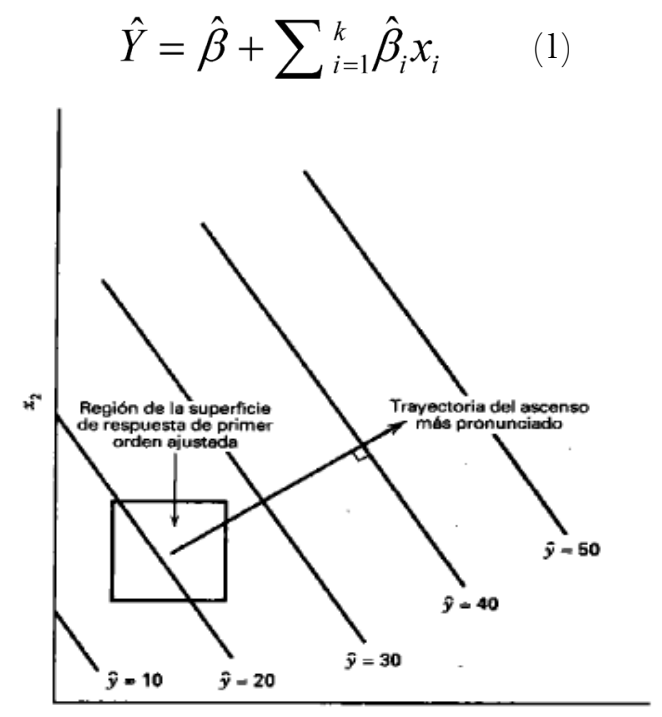

Figure 3. The sequence of the MSR. Source: (Montgomery \& Runger, 1996).

\subsection{FINAL OPTIMIZATION}

When locating ourselves in the previous stages of the experimental region that contains the optimum, in this region, the second-order effects were more significant in absolute value than the first-order effects; this region is appropriately described by second-order mathematical models as shown in equation (2). In this case, we use the hexagonal design of Figure 4. After closing the optimal region, in this stage, the second-order effects are more significant, for which we use the formula.

$$
Y=b_{0}+\sum b_{i} x_{i}+\sum b_{i i} x_{i}^{2}+\Sigma \quad \sum b_{i j} x_{i} x_{j}+\varepsilon
$$

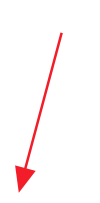

Response variable

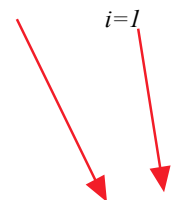

Unknown parameters

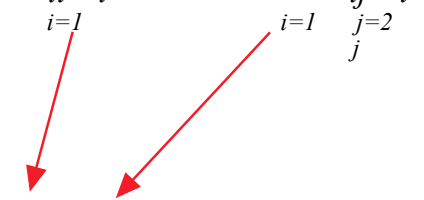

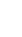

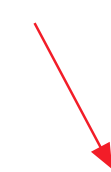

Random error 


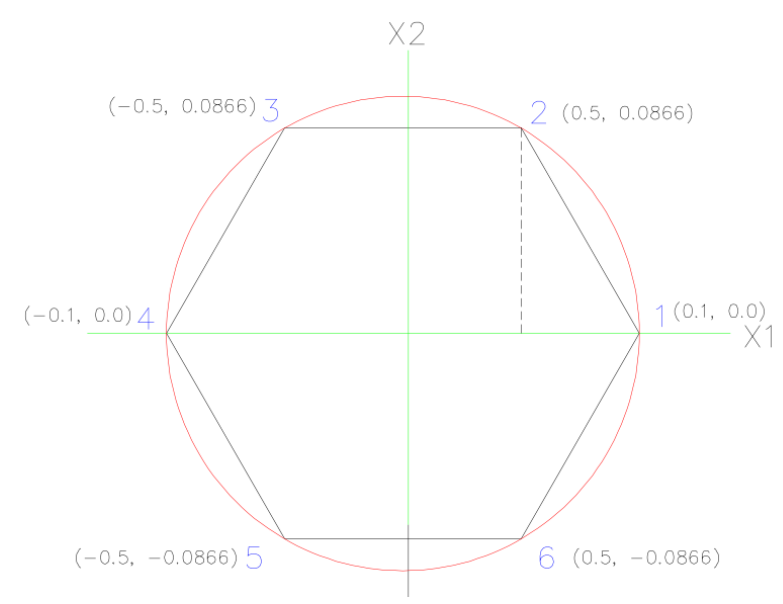

Figure 4. Hexagonal design structure with coded values.

\section{RESULTS}

The beam bending tests carried out at 4, 14, 21, and 28 days provide us with information on the modulus of rupture. It is observed that the mean (41.37) of the module of the ten designs with variable factors concerning the mean of the standard design (36.40) differs in 4.97, the design that grants the highest resistance is the second (44.82) at 28 days of maturation. And for the pattern design, it has a difference of 8.42 .

Table 1. Details of the control.

\begin{tabular}{|c|c|c|c|c|c|c|c|c|c|c|c|c|}
\hline \multirow[b]{2}{*}{ Date Empty } & \multirow[b]{2}{*}{$06 / 01 / 18$} & \multirow[b]{2}{*}{$06 / 01 / 18$} & \multirow[b]{2}{*}{$06 / 01 / 18$} & \multicolumn{4}{|c|}{ Beams $30 \times 30 \times 50$} & \multirow[b]{2}{*}{ 08/01/18 } & \multirow[b]{2}{*}{$08 / 01 / 18$} & \multirow[b]{2}{*}{ 08/01/18 } & \multirow{2}{*}{$\begin{array}{l}\text { Patron } \\
\text { 07/01/18 }\end{array}$} & \multirow[b]{2}{*}{ Mean } \\
\hline & & & & $07 / 01 / 18$ & 07/01/18 & 07/01/18 & 07/01/17 & & & & & \\
\hline Breakdate Date & $03 / 02 / 2018$ & $03 / 02 / 2018$ & $03 / 02 / 2018$ & $04 / 02 / 2018$ & $04 / 02 / 2018$ & $04 / 02 / 2018$ & $04 / 02 / 2017$ & $05 / 02 / 2018$ & $05 / 02 / 2018$ & $05 / 02 / 2018$ & $04 / 02 / 2018$ & \\
\hline READING $\left(\mathrm{Kn} / \mathrm{Cm}^{2}\right)$ & 33.73 & 35.21 & 32.62 & 31.07 & 31.85 & 35.1 & 30.59 & 35.09 & 30.4 & 32.05 & 28.59 & 32.39 \\
\hline READING P $\left(\mathrm{Kg} / \mathrm{Cm}^{2}\right)$ & 3439.45 & 3590.36 & 3326.26 & 3168.21 & 3247.74 & 3579.15 & 3119.26 & 3578.13 & 3099.89 & 3268.14 & 2915.32 & 3302.90 \\
\hline FREE LIGHT L (Cm) & 45 & 45 & 45 & 45 & 45 & 45 & 45 & 45 & 45 & 45 & 45 & 45 \\
\hline AVERAGE WIDTH B (Cm) & 15.30 & 15.40 & 15.20 & 15.40 & 15.50 & 15.40 & 15.30 & 15.50 & 15.40 & 15.30 & 15.60 & 15.39 \\
\hline AVERAGE HEIGHT D $(\mathrm{Cm})$ & 15.50 & 15.30 & 15.30 & 15.50 & 15.30 & 15.40 & 15.20 & 15.40 & 15.60 & 15.30 & 15.20 & 15.36 \\
\hline Edge Distance & 29 & 27 & 17.5 & 24.8 & 26.7 & 24.9 & 17.7 & 20.1 & 29.4 & 25.5 & 28 & 24.6 \\
\hline Distance In middle Third & 11.5 & 9.5 & 0 & 7.3 & 9.2 & 7.4 & 0.2 & 2.6 & 11.9 & 8 & 10.5 & 7.1 \\
\hline BREAKDOWN M $\left(\mathrm{Kg} / \mathrm{Cm}^{2}\right)$ & 42.11 & 44.82 & 42.07 & 38.53 & 40.28 & 44.10 & 39.71 & 43.80 & 37.22 & 41.06 & 36.40 & 41.37 \\
\hline
\end{tabular}

Source: authors' own elaboration. 
The optimal conditions of the controllable, independent variables are Cement $=617.95 \mathrm{~kg} / \mathrm{m}^{3}$ and the fine aggregate $=1816.44 \mathrm{~kg} / \mathrm{m}^{3}$, replacing these values obtained in the coded mathematical model, the maximum strength of the concrete is obtained, which would reach 93\%. By using the MINITAB version 16 software, we elaborated on the optimization graphs for the flexural resistance of rigid pavement concrete.

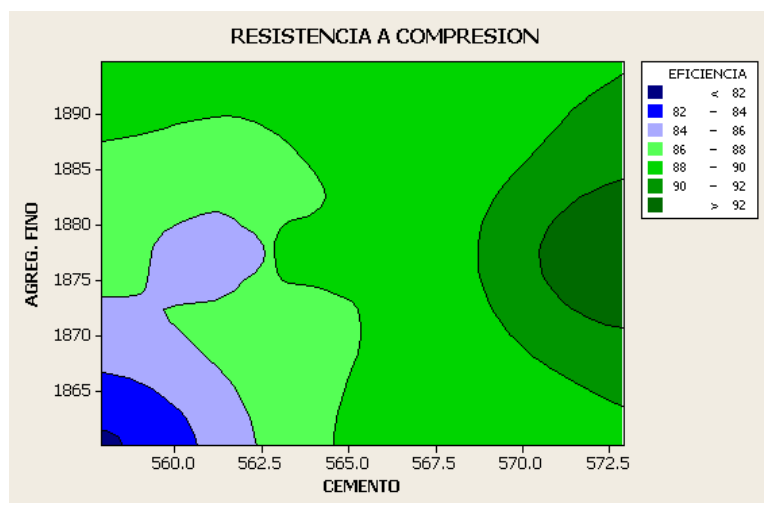

Figure 4. Optimized area for the controlled factors of strength to compressive. Source: authors' own elaboration.

A curve system is observed in the graph, and due to the color distribution, the maximum efficiency is achieved with the maximum level of cement and with a standard level of fine aggregate (dark green color).

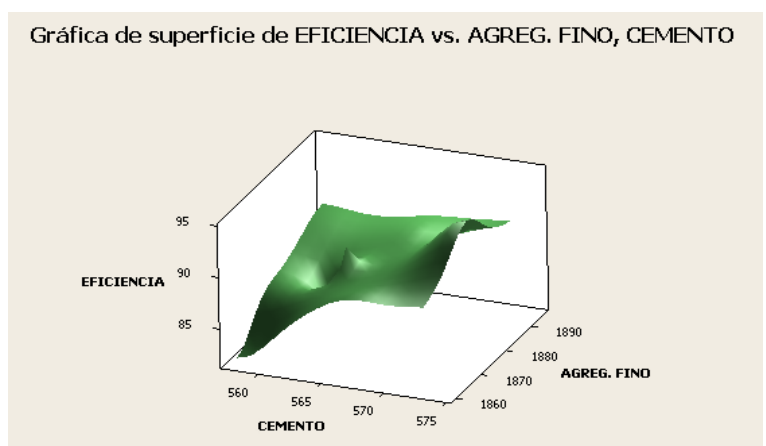

Figure 5. The efficiency of the optimization process regarding fine aggregate and cement. Source: authors' own elaboration. 
In the quadratic effect spatial graph, the contour system is notorious, for the levels generated the maximum efficiency of the concrete resistance is achieved with the maximum levels of cement and the standard level of fine aggregate.

\section{CONCLUSIONS}

The ranges considered concerning direct tensile strength with compressive strength for concretes made with aggregates from the Mantaro river and Portland cement type I were considered between $201 \mathrm{~kg} /$ $\mathrm{cm}^{2}$ and $420 \mathrm{~kg} / \mathrm{cm}^{2}$ and are the ranges of the experiment that they allowed to expand the surface of optimal levels, in terms of the volumes of the materials. Besides, the extension of the working ranges can reduce the consumption of materials, achieving the same resistance that was initially reached.

The Response Surface Methodology is an extremely versatile technique that allows the use of different experimental designs and statistical tools to solve system optimization problems and can be applied to the optimization of a single response or the simultaneous optimization of several responses.

\section{REFERENCES}

Córdova, I. (2012). Proyecto de Investigación Cuantitativa. Editorial San Marcos, p. 77.

Gutiérrez-Pulido, H., \& De la Vara, R. (2008). Análisis y Diseño de Experimentos (2ª ed.). McGraw-Hill.

Huapaya, H. D., Rodriguez, G., \& Esenarro, D. (2020). Comparative analysis of supervised machine learning algorithms for heart disease detection. 3C Tecnología. Glosas de innovación aplicadas a la pyme. Edición Especial, Abril 2020, 233-247. http://doi.org/10.17993/3ctecno.2020. specialissue 5.233-247 
Levy, J., Pandey, B., Chowdhry, B., \& Rodriguez, C. (2020). Prologue: Recent trends in computer science and engineering (RTCSE). 3C Tecnología. Glosas de innovación aplicadas a la pyme. Edición Especial, Abril 2020, 19-25. http://doi.org/10.17993/3ctecno.2020.specialissue5.19-25

Montgomery, D. G., \& Runger, G. G. (1996). Probabilidad y estadística aplicadas a la ingeniería. McGrawHill.

Rosental, M. M., \& Iudin, P. F. (2008). Diccionario Filosófico. Editora Política. http:/ /www.filosofia.org/ urss/dfi1965.htm

Sabino, G. (1986). El proceso de investigación. Editorial Panapo, p. 53. http://paginas.ufm.edu/sabino/ ingles/book/proceso_investigacion.pdf

Sánchez, J., Vega, H., Guzmán, Y., Rodriguez, C., \& Quinto, D. (2020). Data mart design to improve the decision-making process of the after-sales service. Test Engineering and Management, 83, 15481-15494. http://www.testmagzine.biz/index.php/testmagzine/article/view/6539

Soto, B., Vega, H., Guzmán, Y., Rodriguez, G., \& Quinto, D. (2020). Classification algorithm based on machine learning to optimize athletes talent detection. Test Engineering and Management, 83, 13464-13471. https://testmagzine.biz/index.php/testmagzine/article/view/6067

Torre, G. (2004). Tecnología del concreto para ingenieros civiles. LEM-UNI, p. 74. 


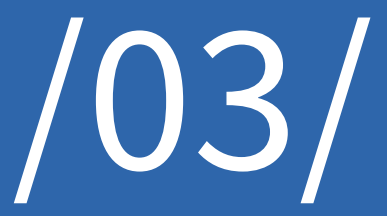




\section{A NOVEL METHOD FOR IMPROVING BIT ERROR RATE IN SENSOR NETWORKS BY USING ORTHOGONAL SPACE TIME BLOCK CODE (OSTBC) CODING}

Richa Tiwari

Assistant Professor, Department of ECE, Vishveshwarya Group of Institution.

Gr. Noida (U.P), (India).

E-mail: richavgi@gmail.com ORCID: https://orcid.org/0000-0001-6924-2217

Deepak Nagaria

Professor, Department of ECE, B.I.E.T,

Jhansi, (U.P), (India).

E-mail: deepaknagaria@bietjhs.ac.in ORCID: https://orcid.org/0000-0002-3043-9765

Rajesh Kumar

Associate Professor, Department of ECE, North Eastern Regional Institute of Science and Technology, Nirjuli, Itanagar,

Arunachal Pradesh, (India).

E-mail: rk@nerist.ac.in ORCID: https://orcid.org/0000-0001-9559-7329

Recepción: 12/05/2020 Aceptación: 08/07/2020 Publicación: 14/09/2020

\section{Citación sugerida:}

Tiwari, R. Nagaria, D., y Kumar, R. (2020). A novel method for improving Bit Error Rate in sensor networks by using Orthogonal Space Time Block Code (OSTBC) coding. 3C Tecnología. Glosas de innovación aplicadas a la pyme, 9(3), 59-75.

https://doi.org/10.17993/3ctecno/2020.v9n3e35.59-75 


\section{ABSTRACT}

For the designing of any network, lifetime and size of the network are the most important parameters in addition to that high data rate and low bit error rate also play an important role in the designing of any sensor network. In this paper, new transmission techniques for the transmission of sensors data has been proposed for sensor networks by combining various modulation and coding techniques into the network transmission. The proposed technique is used to improve the Bit Error Rate performance of the wireless sensor network, in most of the wireless sensor networks ,bits are converted into packets and these packets are transmitted from source to destination during that transmission the quality of physical layer is determined by the Bit Error Rate (BER) and the Packet Delivery Rate (PDR). The physical layer deals with transmission of bits over wireless link the designing constraints of this layer is modulation, diversity and coding. In this paper various modulation, coding and diversity techniques are incorporated into sensor network for reducing Bit Error Rate (BER). The proposed system divides the network into two types of nodes, first one is the sensor nodes, equipped with short distance transmission capability and another one is special nodes that are equipped with modulators and coders for transmitting data over long distance. This proposed system also extended for providing the secured data transmission by the use of various error detection and correction codes.

\section{KEYWORDS}

Bit Error Rate (BER), Orthogonal Space Time Block Code (OSTBC), Internet Of Things (IOT), Orthogonal Transform Division Multiplexing (OTDM), Space Time Coding (STC), Singular Vector Decomposition (SVD). 


\section{INTRODUCTION}

Wireless Sensor Networks (WSNs) are the combination of many tiny sensing elements for transferring data from source to destination using multi-hop transmission. There are a number of applications in which real time monitoring is required so a huge amount of data is collected after the collection of this data various mathematical transformations are required to convert this raw data into useful information. Some applications require security of the data whereas for some applications such as wireless multimedia sensor network major concern is accuracy of the data and high data transfer rate. In agriculture, these networks can provide the report about the growth rate of plants. This can reduce labor work and the cost of production by Zhen, Hong, and Wang (2011) and Sun et al. (2011). However, sensor network has tiny nodes with limited transmission capability and limited battery lifetime so long distance transmission of data is also a challenging task in WSNs. According to an Article "Wireless Channel Propagation Characteristics and modeling research in Rice field sensor networks" by Gao et al. (2018), in wireless sensor network the quality of communication depends upon the condition of the environment where the sensor network is planned to operate for example the attenuation speed in wireless channel propagation is directly related to the development of the rice plant. In this paper author observed that crop plants suffer from different wireless channels effects such as reflection, scattering and diffraction, So various diversity techniques helps us to mitigate these effects in sensor networks. Farhang-Boroujeny and Moradi (2016) showed that, Orthogonal Frequency Division Multiplexing (OFDM) modulation technique played an important role in wireless communication systems due to its ability of high frequency selectivity and achieving high data transfer rate without any Inter Symbol Interference (ISI).

When data travel a long distance then several multipath effects like fading and reflection of signals are also coming into consideration. Diversity techniques are also playing an important role in any communication because these techniques mitigate the effect of multipath fading and shadowing from buildings and objects (Alamouti, 1998). According to a special issue on codes and graphs, in the applications where secure communication is required, the spread spectrum system plays an important role, for the applications 
where the error control is the major requirement then channel encoder and decoder plays an important role because these codes automatically reduce the error in communication. In many applications where size and cost of the antenna is a major limitation, the cooperative transmission method is considered as better approach.

A Wireless sensor network can use generally cooperative relaying for increasing the lifetime of the sensor network. In wireless medium long distance communication requires more power than the short distance communication because after traveling long distance signals become weak.

In the proposed scheme, network nodes are divided into two categories: in first category, nodes are used for sensing and these sensing nodes are having limited transmission capability. In Second category nodes are special modules which have long distance transmission capability where various modulation and coding techniques are used in the designing of these special nodes for improving the Bit Error Rate (BER) performance. If analysis is done on total power consumed, then most of the nodes power are consumed during transmission, so the lifetime of first category automatically increase by the use of these special nodes so introducing these strategies inside the network increase the lifetime of whole sensor network. Aly et al. (2019) proposed a Space-time coding Orthogonal Space Time Block Codes (OSTBC) technique for enhancing the Bit Error Rate (BER) performance, security, increased diversity gain and decrement in the fading effect. Hasna and Alouini (2003) demonstrated that Decode and Forward (DF) protocols performes better at low SNR. Baek and Song (2008) designed and analyzed the performance of cooperative diversity in MIMO-OFDMA system. Jing and Jaferkhani (2009) have analyzed the performance of single and multiple relays and calculate their diversity order. Decode and Forward (DF) protocols are generally used in WSNs where the information bits are detected, decoded and sent forward. Lu, Nikookar and $\mathrm{Xu}$ (2010) demonstrate that decode and forward protocols for reducing channel interference and additive noise at the relay. 
In this paper by using the multiple access techniques data from different sensors are combined together and this data has been sent to the destination by the use of OSTBC encoding and modulation. System becomes more complex in comparison to the normal sensor network at transmission level but the advantage of this system is improvement in Bit Error Rate performance and transmission work is handled by some specific modules so network life time does not depend on the complexity of transmission protocols and all nodes power is not wasted in long distance transmission. Another advantage of using this method of transmission is that the dependency on internet or requirement of replacing the sensor nodes by the small IOT devices has been overcome by installing such type of communication networks (Arroyo et al., 2019).

In Elhabyan and Yagoub (2014), the parameters for judging the performance of any sensor Network are average consumed power, packet delivery rate, network coverage and number of nodes in a particular area.

In WSNs, nodes perform two functions: first sensing the information and second transferring that information to destination. In Castanedo (2013), there are many topologies for arranging the sensor nodes and routing protocols for transferring the sensed information. In Din et al. (2014) \& Han et al. (2014) has proposed many optimization technique for reducing the average consumed power.

The key contribution of this paper is modulation and coding technique are incorporated in the transmission of sensed data. After that simulation has been carried out for different type of modulation such as Frequency shift Keying (FSK) with diversity, Binary Phase shift keying (BPSK) with diversity, BPSK with OSTBC coding and BPSK, BFSK and QAM for different diversity order. The simulation results show the improvement in BER of the network by including the modulation and coding capability in the special nodes on the place of using complex routing algorithms for load sharing. 
The rest paper is arranged as follows. In Section 2, detailed description of the proposed system. System evaluation and simulation results are presented in Section 3. This is followed by the Section 4 that do conclusion and showing the advantages of the proposed technique.

\section{MATERIALS AND METHODS}

The proposed network architecture is shown in Figure 1, which shows that data are coming from multiple sensor nodes, these sensor nodes sensing the data and the data from each sensor node is received in the special node at discrete time intervals. The special nodes perform the data acquisition, modulation and coding. Data received at special node is of two types useful and useless data, useless data include the noise signals and rest of the data is comes into the category of useful data and these are the observations from the detected target. The special node performs data fusion. The techniques which are used for the purpose of fusion are data association techniques (Akkaya \& Younis, 2005), by using that technique data from different sensor nodes are combined together after that special node do modulation and coding for transmitting that data to the destination.

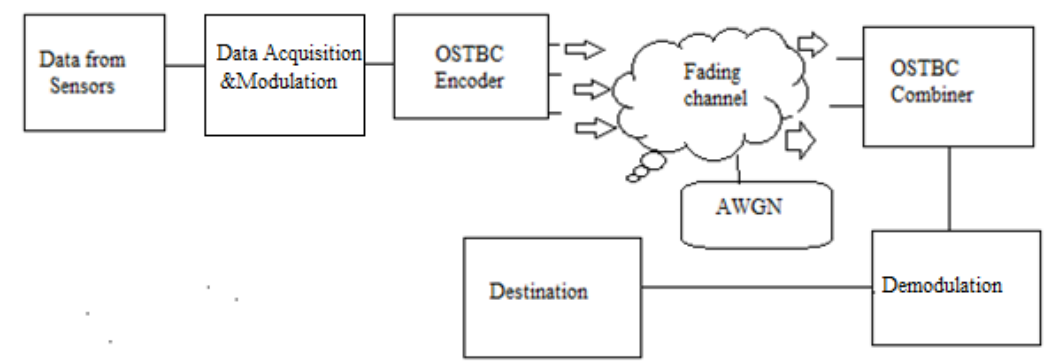

Figure 1. OSTBC coded data transmission from source to destination through Rayleigh fading channel. Source: authors' own elaboration.

The sensing range characteristics are depending on the type of the sensors being used for the purpose of sensing. Such as for PIR sensor it is 20 feet's and for Inductive proximity sensor it is $50 \mathrm{~mm}$ since these sensors are connected to radio transceiver board (Ex CG 2420), transmission range is determined by the transmission power used by the module. The module CC2420 supports the choice of several 
power levels with $0 \mathrm{dbm}$ (eq. $1 \mathrm{mw}$ ) being the maximum power which decides the transmission capability. Transmission range also depends on the environmental condition, obstacles etc. For WSN standard 802.15.4 the communication range is from $20 \mathrm{~m}$ to $30 \mathrm{~m}$ for indoor applications and $75 \mathrm{~m}$ to $100 \mathrm{~m}$ for outdoor applications. After that if we want to increase the communication range or speed of transmission then there are two solutions, first the use of special IOT devices connected to high speed Wi-Fi network, second solution is modulation and coding of the signals.

The incoming data from different sensors are combined together and modulated by any digital modulation techniques such as BPSK, BFSK or QAM etc. after that data is OSTBC encoded and transmitted via fading channel.

The OSTBC Encoder block encodes an input signal sequence using Orthogonal Space Time Block Code. In this case the input signal is sampled because fusion node inside the transmission module select the data at discrete time interval so sampled version of data is ready for transmission on that stage. The OSTBC encoder block supports many OSTBC encoding algorithms that are Depending on the rate and number of transmitting antenna used. In this section, OSTBC codes with 3 transmitting antenna and Rate3/4 is used here. In this paper, 3x2 MIMO is implemented using Alamouti algorithm. A complex orthogonal space-time block code, three consecutive symbols $\mathrm{S}_{1}, \mathrm{~S}_{2}$ and $\mathrm{S}_{3}$ are encoded with the following space-time code word matrix:

$$
\left(\begin{array}{ccc}
S_{1} & S_{2} & S_{3} \\
-S_{2} * & S_{1} * & 0 \\
S_{3} * & 0 & -S_{1} * \\
0 & S_{3} * & -S_{2} *
\end{array}\right)
$$




\subsection{MATHEMATICAL MODELING OF OSTBC ENCODED SIGNAL}

In this section, a brief description about the OSTBC encoding scheme for three transmit antennas and two receiving antennas are given, we are using a Orthogonal Space Time Block Code with three transmit antennas where the rate of this code is $3 / 4$. The input to the OSTBC encoder is a $3 \times 1$ vector signal and the output is a $4 \times 3$ vector signal. A random binary signal is modulated by using Binary phase shift keying (BPSK) after that this signal goes inside an OSTBC coder for transmission over a Rayleigh fading channel. The fading channel model has six independent links due to the three transmitting antennas and two receiving antennas. An additive white Gaussian noise (AWGN) is added at the receiver side and all signals are combined into a single stream for demodulation by using OSTBC combiner.

In the first time instant, antennal transmits $\mathrm{X} 1$, antenna2 transmits $\mathrm{X}_{2}$ and antenna3 transmits $\mathrm{X}_{3}$ while during second time instant, antennal transmits $-\mathrm{X}_{2}^{*}$, antenna 2 transmits $\mathrm{X}_{1}$ *and antenna 3 transmits 0 , in third time instant antennal transmits $\mathrm{X}_{3}{ }^{*}$,antenna 2 transmits 0 and antenna3 transmits $-\mathrm{X}_{1}{ }^{*}$ and in fourth time instant antennal transmits 0 ,antenna 2 transmits $\mathrm{X}_{3}^{*}$ and antenna3 transmits $-\mathrm{X}_{2}^{*}$.

At the receiver side the received signal is given by the following equations:

Received signal at first time slot:

$$
\begin{gathered}
Y_{1}(1)=\left(\begin{array}{lll}
G_{1,1} & G_{2,1} & G_{3,1}
\end{array}\right)\left(\begin{array}{c}
X_{1} \\
X_{2}+n(1) \\
X_{3}
\end{array}\right) \\
Y_{2}(1)=\left(\begin{array}{lll}
G_{1,2} & G_{2,2} & G_{3,2}
\end{array}\right)\left(\begin{array}{c}
X_{1} \\
X_{2}+n(1) \\
X_{3}
\end{array}\right)
\end{gathered}
$$

Received signal at second time slot: 


$$
\begin{gathered}
Y_{1}(2)=\left(\begin{array}{lll}
G_{1,1} & G_{2,1} & G_{3,1}
\end{array}\right)\left(\begin{array}{c}
-X_{2}^{*} \\
X_{1}^{*}+n(2) \\
0
\end{array}\right) \\
Y_{2}(2)=\left(\begin{array}{lll}
G_{1,2} & G_{2,2} & G_{3,2}
\end{array}\right)\left(\begin{array}{c}
-X_{2}^{*} \\
X_{1}^{*}+n(2) \\
0
\end{array}\right)
\end{gathered}
$$

Similarly, we can write down the received signals equation for third and fourth time slot where $\mathrm{n}(3)$ and $\mathrm{n}(4)$ are AWGN , that are added during these slots after that combiner combines all signals and estimate the transmitted signal vector as:

$$
\left(\begin{array}{l}
\hat{x}_{1} \\
\hat{x}_{2} \\
\hat{x}_{3}
\end{array}\right)=\frac{1 \sum_{j=1}^{M}}{\|H\|^{2}}\left(\begin{array}{l}
G_{1, j}^{*} r_{1, j}+G_{2, j}^{*} r_{2, j}-G_{3, j}^{*} r_{3, j} \\
G_{2, j}^{*} r_{1, j}-G_{1, j}^{*} r_{2, j}-G_{3, j}^{*} r_{4, j} \\
G_{3, j}^{*} r_{1, j}+G_{1, j}^{*} r_{3, j}+G_{2, j}^{*} r_{4, j}
\end{array}\right)
$$

Where $\hat{x}_{k}$ represents the $\mathrm{k}^{\text {th }}$ symbol in the OSTBC code matrix $\mathrm{G}_{\mathrm{i}, \mathrm{j}}$ represents the estimate for the channel for $\mathrm{i}^{\text {th }}$ transmitting antenna and $\mathrm{j}^{\text {th }}$ receiving antenna. The values of $\mathrm{i}$ and $\mathrm{j}$ range from 1 to $\mathrm{N}$ (the number of transmitting antennas) and 1 to $\mathrm{M}$ (the number of receiving antennas).

Where $H^{2}=$ summation of channel power per link.

\subsection{BER ANALYSIS}

The Bit Error Rate (BER) of the proposed scheme; the BER is defined as the number of bits in error divided by the total number of transferred bits during the studied time interval so:

$$
B E R=\frac{\text { Total number of bit errors }}{\text { Total number of bits recieved }}
$$


When, we talk about the performance of any sensor network then packet delivery Rate (PDR) is defined as the ratio of the number of packets successfully received by all cluster heads to the number of packets generated so both PDR and BER are related to the information or the data transferred from source to destination. These two parameters are related to the transmission capability of any sensor network so if the BER performance of any network is improved then this improvement automatically reflect in the packet delivery rate or in other words reduction in the BER is the increment in the PDR.

\section{SIMULATION RESULTS}

In this section, the proposed scheme of transmission is compared to the conventional modulation schemes such as BPSK, BFSK and QAM with diversity. Set of architectures are evaluated in terms of modulation with OSTBC coding and modulation with diversity techniques. The performance of these network architectures is discussed in the following subsections. A BPSK modulated system with diversity of 6 is considered and compared this with OSTBC coding of rate $3 / 4$. After that the effect of changing the modulation technique is studied. For simulation a random binary data is created. For the selected OSTBC code the output signal power is $2.25 \mathrm{~W}$ and the channel symbol period for this simulation is 7.5 $\mathrm{e}^{-4}$ sec due to the code rate $3 / 4$. All the parameters used in simulation are collected in Table 1 .

Table 1. Simulation parameters.

\begin{tabular}{|c|c|}
\hline Type of Channel & Rayleigh Fading Channel \\
\hline Type of modulation & BPSK,BFSK,QAM \\
\hline Diversity order & 6 \\
\hline Number of Subcarriers & 1024 \\
\hline Carrier Frequency & $2 \mathrm{Ghz}$ \\
\hline FFT size & 64 \\
\hline Cyclic prefix & $0 \%$ \\
\hline Number of Monte carlo simulation & $2 \mathrm{e} 6$ \\
\hline $\mathrm{E}_{\mathrm{b}} / \mathrm{N}_{0}(\mathrm{~dB})$ & 0 to $30 \mathrm{~dB}$ \\
\hline
\end{tabular}

Source: authors' own elaboration. 


\subsection{EFFECT OF OSTBC CODING}

Figure 2 shows the BER of the proposed technique in comparison to the BPSK with diversity order 6. In OSTBC coded transmission of BPSK modulated signal out of 100000 received bits only 48 bits are in error. The BER reaches about 0.0013 at $9 \mathrm{~dB}$, whereas in diversity transmission this value is 0.0009 at $9 \mathrm{~dB}$. These results showed that there is little bit different in the BER performance of both techniques.

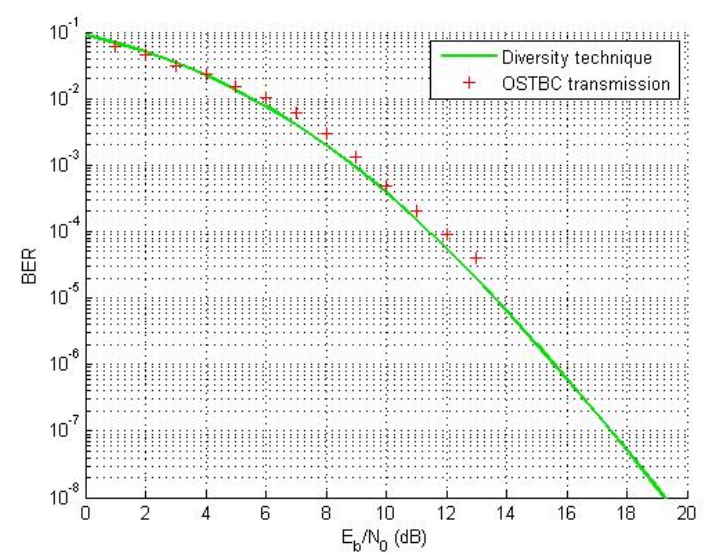

Figure 2. BER of the OSTBC transmission in comparison with diversity transmission of order 6. Source: authors' own elaboration.

\subsection{EFFECT OF MODULATION TECHNIQUE AND DIVERSITY}

Figure 3 shows the effect of changing the modulation technique on the proposed techniques if we use the BFSK in place of BPSK then the performance of OSTBC transmission techniques does not improve the performance of the network in comparison to the Diversity techniques. In the transmission of OSTBC coded FSK, out of 240 received bits 101 bits are in error. BER is 0.0077 at $9 \mathrm{~dB}$, for coherent FSK with diversity order 6 . The BER is 0.0791 for non-coherent FSK with diversity order 6. For OSTBC coded transmission of FSK modulated wave it is too large that is 0.4714 at $9 \mathrm{~dB}$. The BER performance of these technique is shown in Figure 3. 


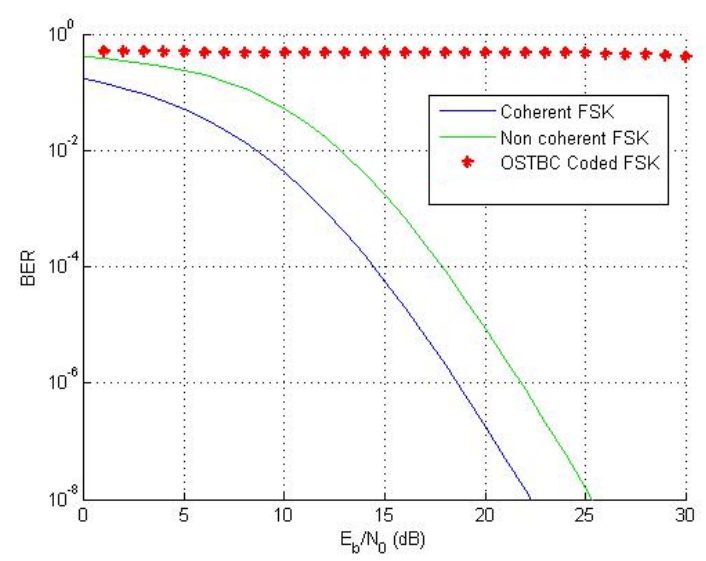

Figure 3. BER of the OSTBC transmission of FSK signal in comparison to the FSK with diversity order 6. Source: authors' own elaboration.

The Performance Comparison of all the transmissions are collected in Table 2.

Table 2. Comparitive Performance.

\begin{tabular}{|c|c|c|c|c|}
\hline Mode of transmission & BER at 3dB & BER at $\mathbf{6 d B}$ & BER at 13 dB & Performance \\
\hline OSTBC coded BPSK & 0.0306 & 0.0100 & $4 \times 10^{-5}$ & Good \\
\hline $\begin{array}{c}\text { OSTBC coded BFSK } \\
\text { Diversity transmission of Coherent } \\
\text { BPSK }\end{array}$ & 0.5104 & 0.4803 & 0.4782 & Goor \\
\hline $\begin{array}{c}\text { Diversity transmission of Coherent } \\
\text { BFSK }\end{array}$ & 0.0344 & 0.0077 & $1.92 \times 10^{-5}$ & Comparatively Low \\
\hline $\begin{array}{c}\text { Diversity transmission of Non- } \\
\text { Coherent BFSK }\end{array}$ & 0.3134 & 0.1952 & $3.84 \times 10^{-4}$ & 0.0084 \\
\hline
\end{tabular}

\subsection{EFFECT OF DIVERSITY ORDER FOR DIFFERENT MODULATION TECHNIQUES}

For agriculture field applications, diversity techniques play an important role to overcome the difficulties arise due to several multipath effects. In wireless sensor network transmission of signal inside the channel depends upon the growth of plants so in such type of situations diversity techniques helps us in estimating the original signal. In that section we also see the effect of using diversity order inside the system. 
For different diversity orders, the calculated BER for different modulation techniques such as Phase shift keying (PSK), Frequency shift keying (FSK) and 4-Qudrature amplitude modulation (4-QAM) is compared in this section which is given in Figures 4, 5, 6. These figure shows how we can improve the BER of any modulation technique by increasing the diversity order. In the agriculture field application, a variable diversity schemes for different growth time will improve the performance of sensor networks.

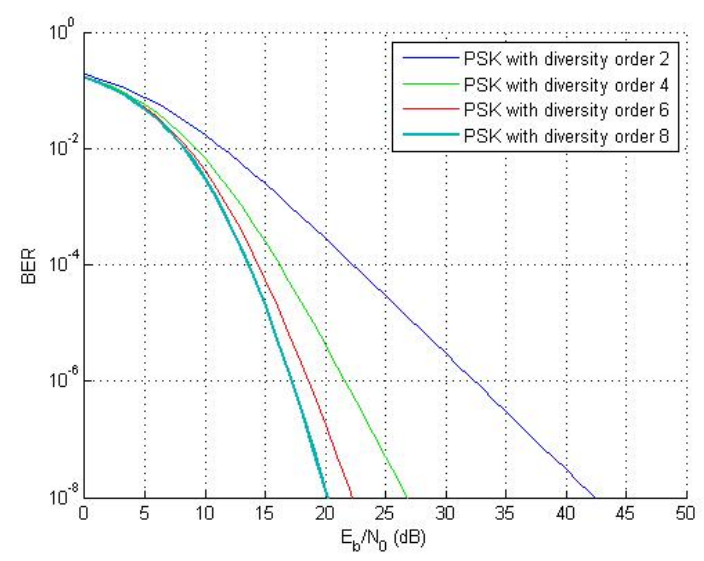

Figure 4. BER of the PSK signal with changing diversity order. Source: authors' own elaboration.

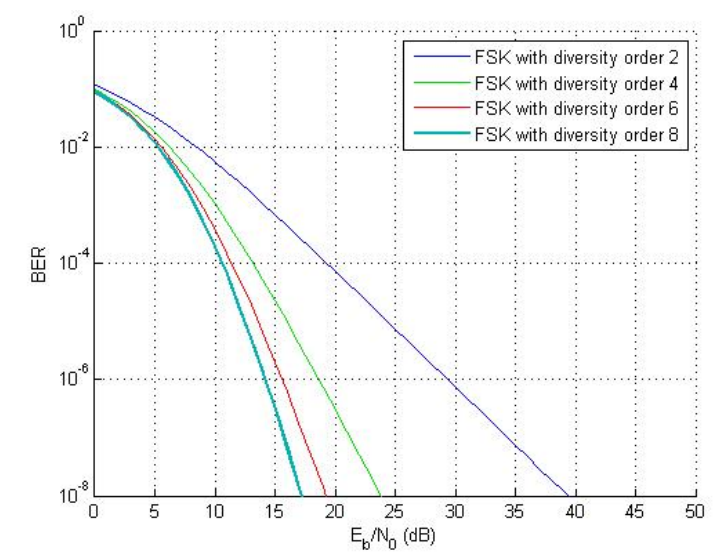

Figure 5. BER of the FSK signal with changing diversity order. Source: authors' own elaboration. 


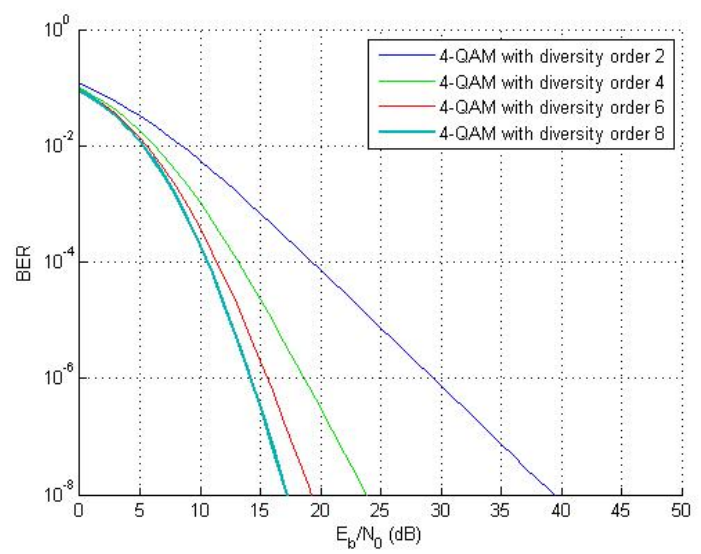

Figure 6. BER of the 4-QAM signal with changing diversity order. Source: authors' own elaboration.

\section{CONCLUSIONS}

An OSTBC coding, diversity and modulation based architecture is proposed for the transmission of data in sensor network. These techniques improved the BER performance of the sensor network. We considered a network with OSTBC coded transmission of BPSK signal. The modulation scheme PSK, FSK and 4-QAM were investigated with different diversity order. For the comparative performance evaluation, we considered the transmission of PSK modulated wave with diversity order 6 and compared that with OSTBC encoded transmission. The proposed transmission shows its superiority over the complex routing algorithm based transmission of sensor network data. In many cases where installing a long antenna is not good choice then by using OSTBC transmission approach, we can achieve the good BER performance. The proposed technique is basically based on sensor fusion or data fusion where data from several sensors are combined together and after that instead of sending a huge amount of data only useful data are send to the destination by means of coding and modulation, the proposed network is applicable and can be practically beneficial for high data rates applications such as wireless multimedia sensor network where transmission quality of video and image signal is the requirement of system. 


\section{ACKNOWLEDGEMENTS}

Richa Tiwari author wishes to express her sincere gratitude to Professor Rajesh Kumar for guiding her throughout the current research work.

\section{REFERENCES}

Akkaya, K., \& Younis, M. (2005). A Survey on routing protocols for wireless sensor network. Ad Hoc Networks, 3(3), 325-349. https://doi.org/10.1016/j.adhoc.2003.09.010

Alamouti, S. M. (1998). A Simple transmit diversity technique for wireless communications. IEEE Fournal on Selected Areas in Communications, 16(8),1451-1458. https://doi.org/10.1109/49.730453

Aly, R. M., Zaki, A., Badawi, W. K., \& Aly, M. H. (2019). Time coding OTDM MIMO System based on singular vector decomposition for 5G Applications. Applied Sciences, 9(13), 2691. https:// doi.org/10.3390/app9132691

Arroyo, P., Herrero, J. L., Suárez, J. I., \& Lozano, J. (2019). Wireless sensor network combined with cloud computing for air quality monitoring. Sensors MDPI, 19(3), 691. https://doi.org/10.3390/ s19030691

Baek, M.-S., \& Song, H.-K. (2008). Cooperative diversity technique for MIMO-OFDM uplink in wireless interactive broadcasting. IEEE Transactions on Consumer Electronics, 54(4), 1627-1634. https://doi.org/10.1109/TCE.2008.4711212

Castanedo, F. (2013). A Review of Data Fusion Techniques. The Scientific world fournal, Article ID 704504. http://dx.doi.org/10.1155/2013/704504 
Din, W., Yahya, S., Taib, M. N., Yassin, A. I., \& Razali, R. (2014). MAP: The new clustering algorithm based on multitier network topology to prolong the lifetime of wireless sensor network. In 2014 IEEE 10th International Colloquium on Signal Processing and its Applications, 173-177. https:// doi.org/10.1109/CSPA.2014.6805742

Elhabyan, R. S., \& Yagoub, M. G. E. (2014). Particle Swarm Optimization Protocol for Clustering in Wireless Sensor Networks: A Realistic Approach. In 2014 IEEE International Conference on Information Reuse and Integration (IRI), Redwood City, CA, USA, 345-350. https://doi.org/10.1109/ IRI.2014.7051910

Farhang-Boroujeny, B., \& Moradi, H. (2016). OFDM inspired waveforms for 5G. IEEE Communications Surveys \&0 Tutorials, 18(4), 2474-2492. https://doi.org/10.1109/COMST.2016.2565566

Gao, Z., Li, W., Zhu, Y., Tian, Y., Pang, F., Cao, W., \& Ni, J. (2018). Wireless Channel Propagation Characteristics and Modeling Research in Rice Field Sensor Networks. Sensors, 18(9), 3116. https://doi.org/10.3390/s18093116

Han, Z., Wu, J., Zhang, J., Liu, L., \& Tian, K. (2014). A General Self-Organized Tree-Based EnergyBalance Routing Protocol for Wireless Sensor Network. IEEE Transactions on Nuclear Science, 61(2), 732-740. https://doi.org/10.1109/TNS.2014.2309351

Hasna, M. O., \& Alouini, M.-S. (2003). Outage probability of multihop transmission over Nakagami fading channels. IEEE Communications Letters, 7(5), 216-218. https://doi.org/10.1109/ LCOMM.2003.812178

Jing, Y., \& Jafarkhani, H. (2009). Single and multiple relay selection schemes and their achievable diversity orders. IEEE Transactions on wireless communications, 8(3), 1414-1423. https://doi. org/10.1109/TWG.2008.080109 
Lu, H., Nikookar, H., \& Xu, T. (2010). OFDM Communication with cooperative Relays. Communications and Networking. Intech Publisher. https://pdfs.semanticscholar. org/54ab/679739d40d0993884795fla682b839bdead9.pdf

Sun, Y. W., Shen, M. X., Lu, M. Z., Lin, X. Z., Xiong, Y. J., \& Liu, L. S. (2011). Research status and prospect of wireless sensor network in agriculture. Acta Agriculturae Zhejiangensis, 23(3), 639-644. https://www.cabdirect.org/cabdirect/abstract/20113305683

Zhen, L. I., Hong, T. S., \& Wang, N. (2011). Review on wireless sensor network technology application in precision agriculture. Fournal of Hunan Agrichltural University, 37(5), 576-580. http://pub. chinasciencejournal.com/JournalofHunanAgrichlturalUniversity/32954.jhtml 


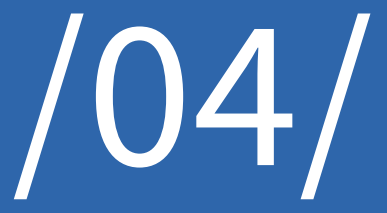




\title{
LEMNA MINOR INFLUENCE IN THE TREATMENT OF ORGANIC POLLUTION OF THE INDUSTRIAL EFFLUENTS
}

\author{
Karina Alvarado \\ Universidad Tecnológica del Perú, Lima, (Perú). \\ E-mail: c10208@utp.edu.pe ORCID: https://orcid.org/0000-0001-7142-4212
}

Doris Esenarro

National University Federico Villarreal, Lima, (Perú). E-mail: desenarro@unfv.edu.pe ORCID: https://orcid.org/0000-0002-7186-9614

Ciro Rodriguez

National University Mayor de San Marcos, Lima, (Perú). E-mail: crodriguezro@unmsm.edu.pe ORCID: https://orcid.org/0000-0003-2112-1349

Wilson Vasquez

Universidad Tecnológica del Perú, Lima, (Perú). E-mail: c18347@utp.edu.pe ORCID: https://orcid.org/0000-0001-7064-028X

Recepción: 08/06/2020 Aceptación: 20/07/2020 Publicación: 14/09/2020

\section{Citación sugerida:}

Alvarado, K., Esenarro, D., Rodriguez, C., y Vasquez, W. (2020). Lemna minor influence in the treatment of organic pollution of the industrial effluents. 3C Tecnología. Glosas de innovación aplicadas a la pyme, 9(3), 77-97. https://doi. org/10.17993/3ctecno/2020.v9n3e35.77-97 


\section{ABSTRACT}

The purpose of the research was to determine the influence of industrial wastewater treatment using the Lemna Minor aquatic plant. Certain varieties of macrophyte plants can absorb or retain various contaminants. Thanks to this, it has been determined that the variety known as Lemna Minor presents this type of property. Three treatment trials were carried out varying the amounts of Lemna Minor (100, 200, and 300g). They are keeping constant the retention time of 10 days that were analyzed at 3, 6, and 10 days after the treatment and with a constant volume of the residual effluent. The results indicate that in terms of the parameters that determine organic contamination, BOD was reduced by (61\%); COD was reduced by $(68 \%)$ and the concentration of total suspended solids by (61\%).

\section{KEYWORDS}

Lemna minor, Organic pollution, Industrial effluents, Wastewater treatment, Macrophyte plants. 


\section{INTRODUCTION}

The effluents from industrial processes coming from dyestuffs present organic matter expressed as chemical oxygen demand (COD), biochemical oxygen demand (BOD), and total suspended solids (TSS). The bathing ratios (BR) in these types of processes comprise 1:7 to 1:12; this leads to the use of large quantities of water. These effluents must be treated before being introduced into the industrial drainage system, to comply with national regulations related to the use of public sewage systems and to avoid contamination of the receptors.

The main benefit of treatment systems with aquatic plants is their low cost of construction and maintenance, as well as their simplicity of operation using an available resource. The presence of a large amount of organic contamination depletes the oxygen in the water, resulting in a decrease in the appropriate conditions for life, producing fermentations that lead to bad odors. The sedimented solids, many of them are toxic because they carry heavy metals such as $\mathrm{Cu}, \mathrm{Mn}, \mathrm{Cd}$ and $\mathrm{Cr}$ (Hoyos et al., 2016).

In this research thesis: Influence of the use of Lemna minor in the treatment of organic contamination of industrial effluents in Cotexsur. The objective of the present work was to determine the influence of the treatment of industrial wastewater from the company Cotexsur, using the aquatic plant Lemna Minor. The research was of an applied type and experimental design with a quantitative approach. The sample was taken considering the convenience and criteria previously evaluated by the types of analysis performed and the treatment proposed (Sun et al., 2020; Walsh et al., 2020).

The theoretical bases that support the research, thus, the advantages of using the Lemna Minor, previous definitions of textile dyes, and parameters for measuring organic contamination. The research was also carried out with Lemna Minor, which has a percentage of removal expressed as COD 72.57 \%, BOD5 $73.36 \%$, total solids $75.21 \%$ and in the first six days of treatment with different masses of $100 \mathrm{~g}, 200 \mathrm{~g}$ of Lemna Minor there is a high percentage of decrease in the concentrations of the mentioned parameters (Hoyos et al., 2016; Li et al., 2020). 


\section{METHOD AND INSTRUMENTS}

The measurements obtained are analyzed (often using statistical methods). The sample was considered according to convenience. The criteria previously evaluated, the types of analysis performed, and the treatment proposed, therefore the sample was non-probabilistic.

The total volume of the sample was 70 liters from the industrial textile effluents. These 70 liters will be divided into 3 types of treatment: 100g, 200g, and 300g of Lemna Minor and each of the treatments with 7 experimental runs (Coronel, 2016).

For the collection of data, as Rodriguez et al. (2020), which made it possible to have better control over the data that allowed the characterization of the effluents from Cotexsur. The following equipment was used to obtain the concentrations of the parameters:

- $\quad$ HI 2210 potentiometer.

- $\quad$ COD digester DRB 200.

- Colorimeter DR 900.

- Equipment for determining BOD.

- Analytical balance NBL 124 E.

- Digital sterilization and drying oven DHG 9023 A.

- Button lid dryer, $150 \mathrm{~mm}$.

The equipment and materials for the analyses were provided by the laboratory of the Universidad Autónoma del Perú, which allowed to determine: PH, Biochemical Oxygen Demand, Chemical Oxygen Demand, and Total Suspended Solids (Jojoa, Rodríguez, \& Cardona, 2015). 


\subsection{ST COLLECTION AND IDENTIFICATION OF LEMNA MINOR}

From the lagoons adjacent to the villa marshes in the Chorrillos district, samples of Lemna Minor were collected to be used in the experimental runs, considering the following conditions:

- Have good pigmentation

- That they do not present an anomaly in any of their parts.

The laboratory where the analyses were carried out has adequate ventilation and sunlight. The temperature and humidity in the experimental runs were:

- Maximum temperature: 210G

- Minimum temperature: $180 \mathrm{C}$

- Humidity: $89 \%$.

\subsection{ND COLLECTION AND ANALYSIS OF SAMPLES OF THE INDUSTRIAL EFFLUENTS OF COTEXSUR}

Wastewater analysis was performed according to standardized methods (Standard Methods for the examination wáter and wastewater, APHA) and instruments, equipment, reagents, which are recommended in the methods. Table 1 details the method or technique applied for each analysis parameter.

Table 1. Health quality monitoring protocol, according to DIGESA (Dirección General de Salud Ambiental).

\begin{tabular}{|c|c|c|}
\hline $\begin{array}{c}\text { Parameters /Dimension of the } \\
\text { dependent variable }\end{array}$ & Method / Technique & Bottle material \\
\hline Biochemical Oxygen Demand & Dilution & Plastic or glass \\
\hline Biochemical Oxygen Demand & Colorimetric & Plastic or glass \\
\hline Total suspended solids & Gravimetric & Plastic or glass \\
\hline PH & Electrometric & Field Determination \\
\hline
\end{tabular}

Source: (Dirección General de Salud Ambiental e Inocuidad Alimentaria (DIGESA), s.f.). 


\section{RESULTS}

\subsection{DESCRIPTIVE}

The results obtained in the development of the research are presented below:

Table 2. Distribution of average BOD concentrations vs. treatment time for $100 \mathrm{~g}$ mass of Lemna Minor.

\begin{tabular}{|c|c|c|}
\hline $\begin{array}{c}\text { Mass Lemna } \\
\text { Minor (g) }\end{array}$ & Time (Days) & $\begin{array}{c}\text { Media DBO } \\
\text { (ppm) }\end{array}$ \\
\hline 100 & 0 & 823 \\
\hline 100 & 3 & 518 \\
\hline 100 & 6 & 389 \\
\hline 100 & 10 & 319 \\
\hline
\end{tabular}

Source: authors' own elaboration.

Table 2 and Figure 1 show the decrease in the average concentration of BOD as a function of time, for a mass in contact of $100 \mathrm{~g}$ of Lemna Minor, the average BOD concentration in the initial time was 823 ppm; when in contact for 3 days the average concentration of BOD decreases to $518 \mathrm{ppm}$; for 6 days of treatment the BOD concentration was 369 ppm, and for 10 days the BOD concentration was reduced to a concentration of 319 ppm. (Esenarro et al., 2020).

Table 3. Distribution of average BOD concentration vs. treatment time for $200 \mathrm{~g}$ Mass of Lemna Minor.

\begin{tabular}{|c|c|c|}
\hline $\begin{array}{c}\text { Mass Lemna } \\
\text { Minor (g) }\end{array}$ & Time (days) & $\begin{array}{c}\text { Media DBO } \\
\text { (ppm) }\end{array}$ \\
\hline 200 & 0 & 823 \\
\hline 200 & 3 & 468 \\
\hline 200 & 6 & 346 \\
\hline 200 & 10 & 389 \\
\hline
\end{tabular}

Source: authors' own elaboration. 
In Table 3, we can see the decrease in the average concentration of the biochemical oxygen demand as a function of time. For a mass in contact of $200 \mathrm{~g}$ of Lemna Minor, the average concentration for the initial contact time was 823 ppm; when in contact for 3 days, the average concentration of the biochemical oxygen demand decreased to 468 ppm; for 6 days of treatment, the BOD concentration decrease to $346 \mathrm{ppm}$ for 10 days, the BOD concentration was reduced to $389 \mathrm{ppm}$.

Table 4. Distribution of average BOD concentration vs. treatment time for $300 \mathrm{~g}$ mass of Lemna Minor.

\begin{tabular}{|c|c|c|}
\hline $\begin{array}{c}\text { Mass Lemna } \\
\text { Minor (g) }\end{array}$ & Time (days) & $\begin{array}{c}\text { Media DBO } \\
\text { (ppm) }\end{array}$ \\
\hline 300 & 0 & 823 \\
\hline 300 & 3 & 429 \\
\hline 300 & 6 & 331 \\
\hline 300 & 10 & 533 \\
\hline
\end{tabular}

Source: authors' own elaboration.

In Table 4, the decrease in time-averaged BOD concentrations for a contact mass of $300 \mathrm{~g}$ of Lemna Minor is shown. The average concentration for the initial treatment time was $823 \mathrm{ppm}$. This, when in contact for 3 days, the average concentration of BOD decreases to $429 \mathrm{ppm}$. For 6 days of contact, the BOD concentration was $331 \mathrm{ppm}$, and for 10 days, the BOD concentration was 533 ppm.

Table 5. Distribution of average BOD concentration vs. treatment time and mass of Lemna Minor.

\begin{tabular}{|c|c|c|c|c|}
\hline Mass $\mathbf{( g )}$ & Time (days) & $\begin{array}{c}\text { BOD average } \\
\mathbf{( p p m )}\end{array}$ & $\begin{array}{c}\text { Standard } \\
\text { deviation }\end{array}$ & $\mathbf{N}$ \\
\hline & 0 days & 833,57 & 26,018 & 7 \\
\hline \multirow{2}{*}{100} & 3 days & 517,71 & 34,028 & 7 \\
& 6 days & 389,43 & 29,205 & 7 \\
& 10 days & 318,57 & 20,354 & 28 \\
\hline
\end{tabular}




\begin{tabular}{|c|c|c|c|c|}
\hline Mass $\mathbf{( g )}$ & Time (days) & $\begin{array}{c}\text { BOD average } \\
\mathbf{( p p m )}\end{array}$ & $\begin{array}{c}\text { Standard } \\
\text { deviation }\end{array}$ & $\mathbf{N}$ \\
\hline & 0 days & 822,57 & 26,018 & 7 \\
\hline \multirow{2}{*}{200} & 3 days & 468,29 & 34,898 & 7 \\
& 6 days & 345,57 & 33,125 & 7 \\
\hline & 10 days & 388,57 & 30,237 & 28 \\
\hline & Total & 506,25 & 193,563 & 7 \\
\hline & 0 days & 822,57 & 26,018 & 7 \\
\hline & 3 days & 429,43 & 27,367 & 7 \\
\hline & 6 days & 331,43 & 15,736 & 28 \\
\hline & 10 days & 532,86 & 17,995 & 21 \\
\hline & Total & 529.07 & 188,367 & 21 \\
\hline & 0 days & 822,57 & 24,683 & 21 \\
\hline & 3 days & 471,81 & 48,041 & 21 \\
\hline
\end{tabular}

Source: authors' own elaboration.

Table 5 shows the averages of BOD concentration versus treatment time at different masses of Lemna Minor. The results obtained according to ANOVA show that the greatest decrease in BOD concentration was for a mass of $100 \mathrm{~g}$ of Lemna Minor and 10 days of treatment with a decrease in the concentration of biochemical oxygen demand from $823 \mathrm{ppm}$ to $319 \mathrm{ppm}$.

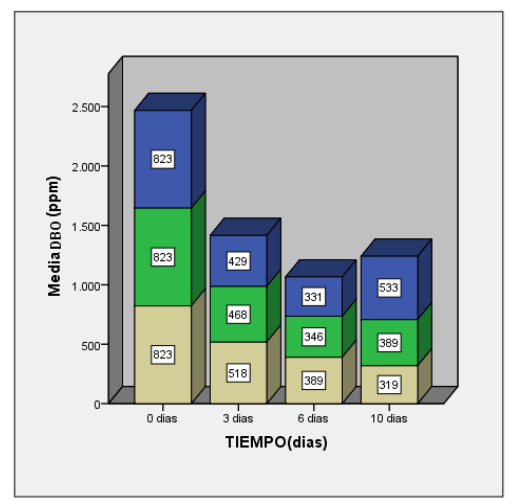

ASA(gramos)

Q300 gramos
$\square 00$ gramos
$\square 100$ gramos

然

Figure 1. Average BOD concentration levels vs. treatment time and mass of Lemna Minor. Source: authors' own elaboration. 
Figure 1 shows the decrease in average BOD concentration for a treatment time of 10 days and mass of 100 Lemna Minor $\mathrm{g}$ from 823 to $319 \mathrm{ppm}$. With a treatment of $200 \mathrm{~g}$ of Lemna Minor, the BOD concentration decreases in 6 days from 823 ppm to $346 \mathrm{ppm}$ and then increases in 10 days to $389 \mathrm{ppm}$, the same behavior is obtained for treatment with $300 \mathrm{~g}$ of Lemna Minor. The BOD concentration decreases in 6 days from 823 ppm to 331 ppm, concluding: a better treatment of organic contamination expressed as BOD is obtained with a mass of $100 \mathrm{~g}$ of Lemna Minor.

Table 6. Distribution of average COD concentrations vs. treatment time for a mass of $100 \mathrm{~g}$ of Lemna Minor.

\begin{tabular}{|c|c|c|}
\hline $\begin{array}{c}\text { Mass Lemna } \\
\text { Minor (g) }\end{array}$ & Time (days) & $\begin{array}{c}\text { DQO average } \\
\text { (ppm) }\end{array}$ \\
\hline 100 & 0 & 1747 \\
\hline 100 & 3 & 1062 \\
\hline 100 & 6 & 749 \\
\hline 100 & 10 & 554 \\
\hline
\end{tabular}

Source: authors' own elaboration.

In Table 6, we can see the decrease in the average concentration of chemical oxygen demand as a function of time for a treatment with a mass of Lemna Minor of $100 \mathrm{~g}$ of Lemna Minor, the average concentration in the initial time was 1747 ppm; when being in contact for a period of 3 days, the average concentration of chemical oxygen demand decreases to 1062 ppm; for 6 days of contact the COD concentration was 749 ppm, and in 10 days a COD concentration of 554 ppm is obtained, obtaining a considerable reduction.

Table 7. Distribution of average COD concentrations vs. treatment time for $200 \mathrm{~g}$ mass of Lemna Minor.

\begin{tabular}{|c|c|c|}
\hline $\begin{array}{c}\text { Mass Lemna } \\
\text { Minor } \mathbf{( g )}\end{array}$ & Time (days) & $\begin{array}{c}\text { DQO average } \\
\text { (ppm) }\end{array}$ \\
\hline 200 & 0 & 1747 \\
\hline 200 & 3 & 913 \\
\hline 200 & 6 & 603 \\
\hline 200 & 10 & 684 \\
\hline
\end{tabular}

Source: authors' own elaboration. 
Table 7 show the decrease in the time-averaged concentrations of the chemical oxygen demand for a treatment with a Lemna Minor mass of $200 \mathrm{~g}$ Lemna Minor. The average concentration in the initial time was 1747 ppm; when in contact with Lemna Minor for 3 days the average concentration of the chemical oxygen demand decreases to 913 ppm; for 6 days of contact with Lemna Minor the COD concentration was $603 \mathrm{ppm}$, and for 10 days an increase in the COD concentration to $684 \mathrm{ppm}$ is obtained.

Table 8. Distribution of average COD concentrations vs. treatment time for $300 \mathrm{~g}$ mass of Lemna Minor.

\begin{tabular}{|c|c|c|}
\hline $\begin{array}{c}\text { Mass Lemna Minor } \\
(\mathbf{g})\end{array}$ & Time (days) & $\begin{array}{c}\text { DQO average } \\
\text { (ppm) }\end{array}$ \\
\hline 300 & 0 & 1747 \\
\hline 300 & 3 & 1046 \\
\hline 300 & 6 & 839 \\
\hline 300 & 10 & 1250 \\
\hline
\end{tabular}

Source: authors' own elaboration.

Table 8 show the decrease in the time-averaged concentrations of chemical oxygen demand for a contact mass of $300 \mathrm{~g}$ of Lemna Minor. The average concentration in the initial time was $1747 \mathrm{ppm}$; when in contact with Lemna Minor for a period of 3 days the average concentration of the chemical oxygen demand decreases to 1046 ppm; for 6 days of contact the COD concentration was 839 ppm, and for 10 days an increase in the COD concentration to $1250 \mathrm{ppm}$ is obtained.

Table 9. Distribution of average COD concentrations vs. treatment time and mass of Lemna Minor.

\begin{tabular}{|c|c|c|c|c|}
\hline MASA (gramos) & TIEMPO (días) & DQO average (ppm) & Desviación estándar & N \\
\hline & 0 días & 1747,14 & 85,968 & 7 \\
\hline \multirow{2}{*}{100 gramos } & 3 dias & 1061,71 & 59,905 & 7 \\
\hline & 6 dias & 748,57 & 86,877 & 7 \\
\hline
\end{tabular}




\begin{tabular}{|c|c|c|c|c|}
\hline MASA (gramos) & TIEMPO (días) & DQO average (ppm) & Desviación estándar & $\mathbf{N}$ \\
\hline \multirow{5}{*}{200 gramos } & 0 días & 1747,14 & 85,968 & 7 \\
\hline & 3 dias & 912,86 & 59,362 & 7 \\
\hline & 6 dias & 602,86 & 51,223 & 7 \\
\hline & 10 dias & 684,29 & 61,606 & 7 \\
\hline & Total & 986,79 & 465,936 & 28 \\
\hline \multirow{5}{*}{300 gramos } & 0 días & 1747,14 & 85,968 & 7 \\
\hline & 3 dias & 1045,71 & 58,838 & 7 \\
\hline & 6 dias & 838,57 & 49,473 & 7 \\
\hline & 10 dias & 1250 & 96,609 & 7 \\
\hline & Total & 1220,36 & 350,571 & 28 \\
\hline \multirow{5}{*}{ Total } & 0 días & 1747,14 & 81,556 & 21 \\
\hline & 3 dias & 1006,76 & 88,581 & 21 \\
\hline & 6 dias & 730 & 117,004 & 21 \\
\hline & 10 dias & 829,52 & 318,535 & 21 \\
\hline & Total & 1078,36 & 438,263 & 84 \\
\hline
\end{tabular}

Source: authors' own elaboration.

In Table 9 the distribution of average COD concentrations vs. treatment time at different masses of Lemna Minor is shown. The results obtained according to ANOVA were determined that the best reduction of COD concentration was with a mass of $100 \mathrm{~g}$ of Lemna Minor and 10 days of treatment; the chemical oxygen demand was reduced from $1747 \mathrm{ppm}$ to $554 \mathrm{ppm}$. 


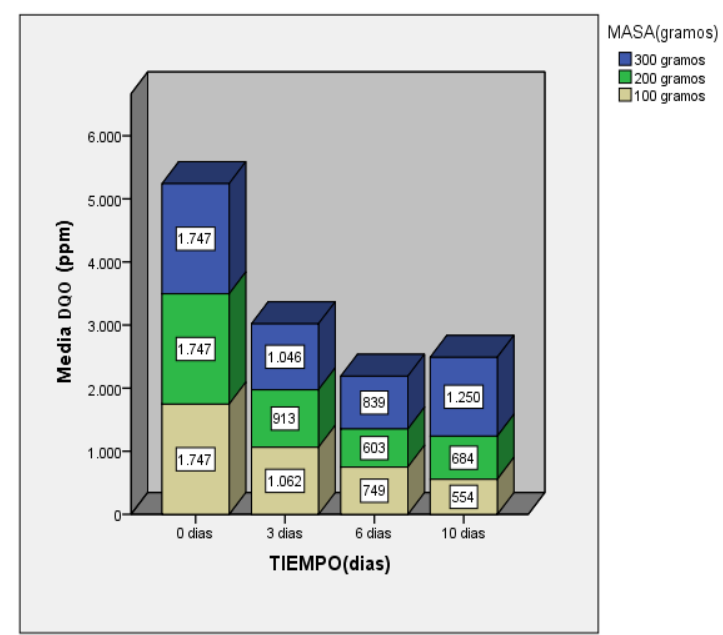

Figure 2. Average COD concentration levels vs. treatment time and mass of Lemna Minor. Source: authors' own elaboration.

Figure 2 shows the most significant reduction in average COD concentration for a treatment time of 10 days and a mass of $100 \mathrm{~g}$ from 1747 to $554 \mathrm{ppm}$. With a treatment of $200 \mathrm{~g}$ of Lemna Minor, the COD concentration decreases in 6 days from 1747 to $603 \mathrm{ppm}$ and then increases in 10 days to $684 \mathrm{ppm}$. The same behavior is obtained for treatment with $300 \mathrm{~g}$ of Lemna Minor, the COD concentration decreases in 6 days from 1747 to $639 \mathrm{ppm}$, concluding that a better treatment of organic contamination expressed as COD is obtained with a mass of $100 \mathrm{~g}$ of Lemna Minor.

Table 10. Distribution of TSS concentration averages vs. treatment time for a mass of $100 \mathrm{~g}$ of Lemna Minor.

\begin{tabular}{|c|c|c|}
\hline $\begin{array}{c}\text { Mass Lemna } \\
\text { Minor (g) }\end{array}$ & Time (days) & Media SST (ppm) \\
\hline 100 & 0 & 124 \\
\hline 100 & 3 & 82 \\
\hline 100 & 6 & 60 \\
\hline 100 & 10 & 48 \\
\hline
\end{tabular}

Source: authors' own elaboration. 
In Table 10, the decrease of the average concentrations as a function of time of the total suspended solids for a treatment with a mass of $100 \mathrm{~g}$ of Lemna Minor, the average concentration of TSS initially was $124 \mathrm{ppm}$; when being in contact for 3 days, the average concentration of TSS decreases to $82 \mathrm{ppm}$; for 6 days of contact, the concentration of TSS $60 \mathrm{ppm}$ and in 10 days a concentration of TSS at 48 ppm is obtained, obtaining a considerable reduction.

Table 11. Distribution of TSS concentration averages vs. treatment time for $200 \mathrm{~g}$ mass of Lemna Minor.

\begin{tabular}{|c|c|c|}
\hline $\begin{array}{c}\text { Mass Lemna } \\
\text { Minor (g) }\end{array}$ & Time (days) & Media SST (ppm) \\
\hline 200 & 0 & 124 \\
\hline 200 & 3 & 86 \\
\hline 200 & 6 & 63 \\
\hline 200 & 10 & 78 \\
\hline
\end{tabular}

Source: authors' own elaboration.

Table 11 show the decrease in the time-averaged concentrations of total suspended solids for treatment with $200 \mathrm{~g}$ Lemna Minor. The average concentration of TSS initially was $124 \mathrm{ppm}$; when in contact for 3 days, the average concentration of TSS decreases to $86 \mathrm{ppm}$. On the other hand, for 6 days of contact, the concentration of TSS is 63 ppm, and in 10 days, a concentration of TSS at 78 ppm is obtained, obtaining a considerable reduction.

Table 12. Distribution of TSS concentration averages vs. treatment time for $300 \mathrm{~g}$ mass of Lemna Minor.

\begin{tabular}{|c|c|c|}
\hline $\begin{array}{c}\text { Mass Lemna } \\
\text { Minor } \mathbf{( g )}\end{array}$ & Time (days) & $\begin{array}{c}\text { Media SST } \\
\text { (ppm) }\end{array}$ \\
\hline 300 & 0 & 124 \\
\hline 300 & 3 & 75 \\
\hline 300 & 6 & 51 \\
\hline 300 & 10 & 99 \\
\hline
\end{tabular}

Source: authors' own elaboration. 
In Table 12 the decrease of the average concentrations as a function of time of the total suspended solids for a treatment with a mass of $300 \mathrm{~g}$ of Lemna Minor, the average concentration of TSS initially was 124 ppm; when being in contact for 3 days the average concentration of TSS decreases to 75 ppm; for 6 days of contact the concentration of TSS to $51 \mathrm{ppm}$ and in 10 days a concentration of TSS to $99 \mathrm{ppm}$ is obtained, obtaining an increase.

Table 13. Distribution of TSS concentration averages vs. treatment time and mass of Lemna Minor.

\begin{tabular}{|c|c|c|c|c|}
\hline MASA (gramos) & TIEMPO (días) & Media SST (ppm) & Desviación estándar & $\mathbf{N}$ \\
\hline \multirow{5}{*}{100 gramos } & 0 días & 123,71 & 8,361 & 7 \\
\hline & 3 dias & 82,29 & 6,157 & 7 \\
\hline & 6 dias & 60 & 6,733 & 7 \\
\hline & 10 dias & 48,43 & 4,685 & 7 \\
\hline & Total & 78,61 & 29,93 & 28 \\
\hline \multirow{5}{*}{200 gramos } & 0 días & 123,71 & 8,361 & 7 \\
\hline & 3 dias & 85,86 & 6,283 & 7 \\
\hline & 6 dias & 63 & 3,367 & 7 \\
\hline & 10 dias & 77,71 & 4,923 & 7 \\
\hline & Total & 87,57 & 23,524 & 28 \\
\hline \multirow{5}{*}{300 gramos } & 0 días & 123,71 & 8,361 & 7 \\
\hline & 3 dias & 74,71 & 7,544 & 7 \\
\hline & 6 dias & 51,14 & 3,288 & 7 \\
\hline & 10 dias & 98,57 & 2,507 & 7 \\
\hline & Total & 87,04 & 28,083 & 28 \\
\hline \multirow{5}{*}{ Total } & 0 días & 123,71 & 7,932 & 21 \\
\hline & 3 dias & 80,95 & 7,934 & 21 \\
\hline & 6 dias & 58,05 & 6,845 & 21 \\
\hline & 10 dias & 74,9 & 21,445 & 21 \\
\hline & Total & 84,4 & 27,295 & 84 \\
\hline
\end{tabular}

Source: authors' own elaboration. 


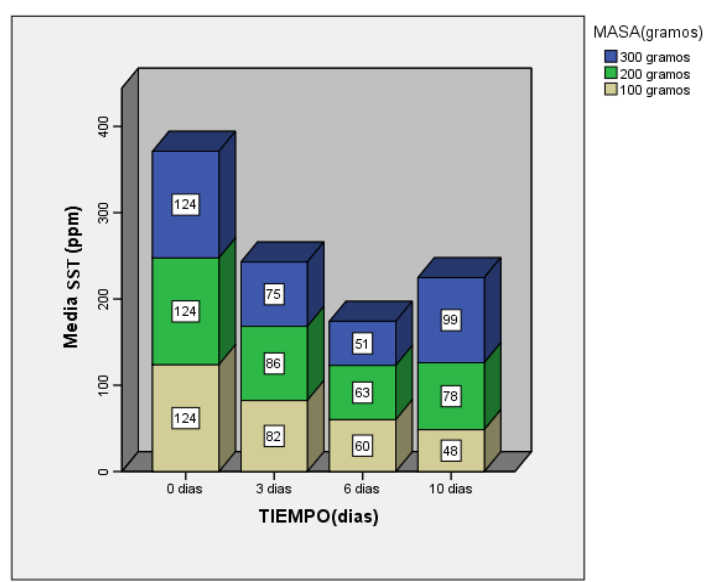

Figure 3. Average TSS concentration levels vs. treatment time and Lemna Minor mass. Source: authors' own elaboration.

In Figure 3, it can be seen that the most significant reduction in average TSS concentration is achieved with a treatment time of 10 days and a mass of $100 \mathrm{~g}$ of 124 to $48 \mathrm{ppm}$. With a treatment of Lemna Minor of $200 \mathrm{~g}$, the concentration of TSS decreases in 6 days from 124 to $63 \mathrm{ppm}$. It then increases in 10 days to $78 \mathrm{ppm}$; the same behavior is obtained for treatment with $300 \mathrm{~g}$ of Lemna Minor, the concentration of TSS decreases in 6 days from 124 to $51 \mathrm{ppm}$ and at 10 days increases to $99 \mathrm{ppm}$, concluding that a better treatment of organic contamination expressed as TSS is obtained with a mass of $100 \mathrm{~g}$ of Lemna Minor.

Table 14. Distribution of $\%$ Average removal of BOD concentration vs. treatment time for a mass of $100 \mathrm{~g}$ of Lemna Minor.

\begin{tabular}{|c|c|c|}
\hline $\begin{array}{c}\text { Masa Lemna } \\
\text { Minor }(\mathbf{g})\end{array}$ & Media DBO & \% Remotion \\
\hline 100 & $0-3$ días & 37 \\
\hline 100 & $0-6$ días & 53 \\
\hline 100 & $0-10$ días & 61 \\
\hline
\end{tabular}

Source: authors' own elaboration. 


\section{\% Average removal DBO (100 g)}

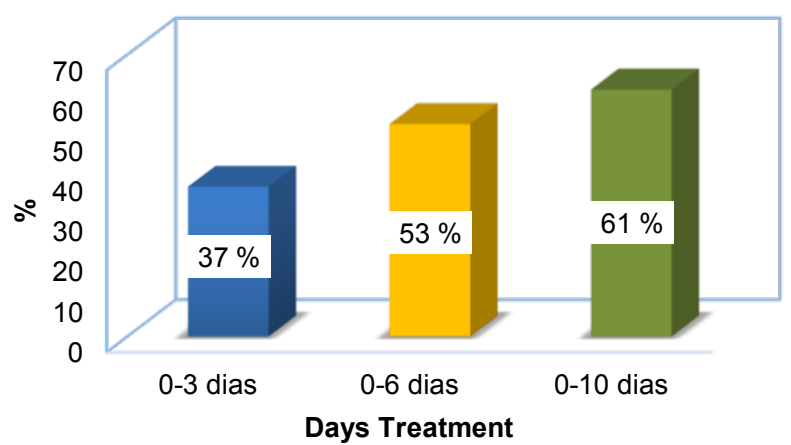

Figure 4. Average \% BOD removal concentration vs. treatment time for $100 \mathrm{~g}$ mass of Lemna Minor. Source: authors' own elaboration.

Table 14 and Figure 4 show the average levels of \% removal of BOD BOD concentration vs. treatment time for a treatment mass of $100 \mathrm{~g}$ of Lemna Minor. On the third day of treatment, $37 \%$, on the sixth day, $53 \%$, and the tenth day of treatment, $61 \%$ of average BOD removal was obtained. It is concluded that for the treatment with a mass of $100 \mathrm{~g}$ of Lemna Minor, the maximum removal of BOD is obtained at the tenth day of treatment.

Table 15. Distribution of $\%$ Average removal of BOD concentration vs. treatment time for a mass of $200 \mathrm{~g}$ of Lemna Minor.

\begin{tabular}{|c|c|c|}
\hline $\begin{array}{c}\text { Mass Lemna } \\
\text { Minor }(\mathbf{g})\end{array}$ & Media DBO & \% Remotion \\
\hline 200 & $0-3$ días & 43 \\
\hline 200 & $0-6$ días & 58 \\
\hline 200 & $0-10$ días & 53 \\
\hline
\end{tabular}

Source: authors' own elaboration.

Table 15 show the average levels of \% removal of BOD concentration vs. treatment time for a treatment mass of $200 \mathrm{~g}$ of Lemna Minor. On the third day of treatment, $43 \%$, on the sixth day of $58 \%$, and the tenth day of treatment, $53 \%$ of average BOD removal was obtained. It is concluded that for the 
treatment with a mass of $200 \mathrm{~g}$ of Lemna Minor, the maximum removal of BOD is obtained on the sixth day of treatment.

Table 16. Distribution of average removal of BOD concentration vs. treatment time for a $300 \mathrm{~g}$ mass of Lemna Minor.

\begin{tabular}{|c|c|c|}
\hline $\begin{array}{c}\text { Mass Lemna Minor } \\
(\mathbf{g})\end{array}$ & Media DBO & \% Remotion \\
\hline 300 & $0-3$ días & 48 \\
\hline 300 & $0-6$ días & 60 \\
\hline 300 & $0-10$ días & 35 \\
\hline
\end{tabular}

Source: authors' own elaboration.

\section{$\%$ media remotion DBO (300 g)}

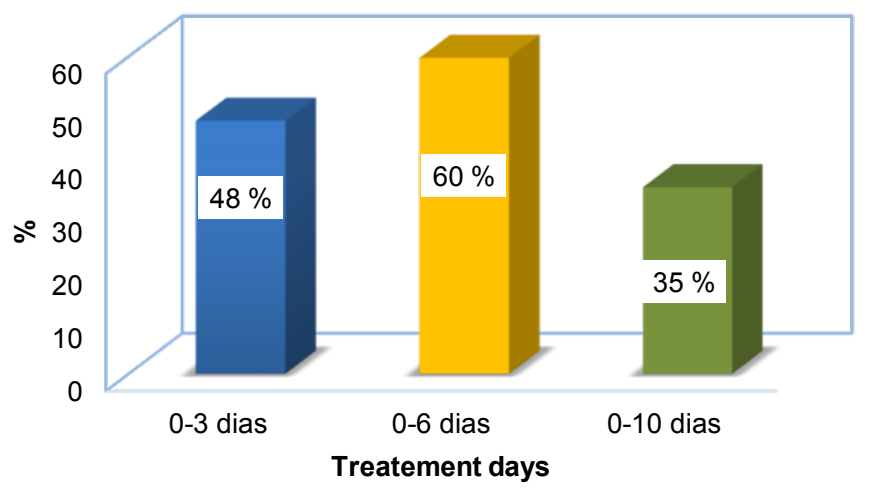

Figure 5. Average \% removal of BOD concentration vs. treatment time for $300 \mathrm{~g}$ mass of Lemna Minor. Source: authors' own elaboration.

In Table 16 and Figure 5, the average levels of \% removal of concentration of the biochemical demand of oxygen BOD versus the time of treatment for a treatment mass of $300 \mathrm{~g}$ of Lemna Minor is observed, obtaining $48 \%$ on the third day of treatment, $60 \%$ on the sixth day and $35 \%$ on the tenth day of treatment of average removal of BOD. It is concluded that for the treatment with a mass of $300 \mathrm{~g}$ of Lemna Minor, the maximum removal of BOD is obtained on the sixth day of treatment. 


\section{DISCUSSION}

Sarango, Sánchez and Landívar (2016), in their experimental design and applied type, designed 2 biofilters of $68 \mathrm{~L}$ capacity each. In contrast to the present research, both investigations were carried out with Lemna Minor applied to industrial effluents, in which there is a percentage of removal expressed as COD $72.57 \%$, BOD5 $73.36 \%$, total solids $75.21 \%$ and in the first six days of treatment with different masses of $100 \mathrm{~g}, 200 \mathrm{~g}$ of Lemna Minor there is a high percentage of decrease in the concentrations of the parameters mentioned expressed as organic matter. In the treatment with $300 \mathrm{~g}$ of Lemna Minor, there is a decrease but not in the same proportion. From the sixth day of treatment, there is a minimum reduction or increase depending on the masses of Lemna Minor due to a stage of withering, which decreases its capacity of assimilation.

But in both, it is concluded that there is a decrease of COD, BOD5, and TSS in the treatment of industrial effluents with Lemna Minor.

\section{CONCLUSION}

Of the three treatments of $100 \mathrm{~g}, 200 \mathrm{~g}$, and $300 \mathrm{~g}$ of Lemna Minor, it is observed that the parameter of BOD obtains a greater removal at 10 days of treatment with a mass of $100 \mathrm{~g}$ Lemna Minor and 6 days with $300 \mathrm{~g}$ of Lemna Minor.

For the DQO in the treatments with $100 \mathrm{~g}, 200 \mathrm{~g}$, and $300 \mathrm{~g}$ of Lemna Minor, a greater removal is obtained to the 10 days of treatment with a mass of $100 \mathrm{~g}$ of Lemna Minor and the 6 days with $200 \mathrm{~g}$ of Lemna Minor.

For TSS in the treatments of $100 \mathrm{~g}, 200 \mathrm{~g}$, and $300 \mathrm{~g}$ of Lemna Minor, there is a greater removal at 10 days of treatment with a mass of $100 \mathrm{~g}$ of Lemna Minor and at 6 days with $300 \mathrm{~g}$ of Lemna Minor. 
From the analysis of the parameters that measure organic contamination such as BOD, COD, and TSS versus contact time we found that there is an inverse relationship, as contact time increases these parameters decrease, but in the tests carried out with 200 and $300 \mathrm{~g}$ it increases from the 6th day, this occurs due to the increase of Lemna Minor that dies during the treatment.

\section{REFERENCES}

Coronel, E. (2016). Eficiencia del Jacinto de agua (Eichhornia Crassipes) y Lenteja de agua (Lemna Minor) en el tratamiento de las aguas residuales de la Universidad Nacional Toribio. Facultad de Ingeniería Civil y ambiental. Universidad Nacional “Toribio Rodríguez de Mendoza de Amazonas”. http:// repositorio.untrm.edu.pe/bitstream/handle/UNTRM/657/EFICIENCIA\%20DEL\%20 JACINTO $\% 20$ DE $\% 20$ AGUA.pdf? sequence $=1$ \&isAllowed $=\mathrm{y}$

Dirección General de Salud Ambiental e Inocuidad Alimentaria (DIGESA). (s.f.). Ministerio de Salud. Perú. http://www.digesa.minsa.gob.pe/

\section{Esenarro, D., Rodriguez, G., Aquije, G., Obregon, N., Anicama, L., \& Arguedas, G.} (2020). Cable Car with Water Collection for Afforestation of the Solar Hill in Chorrillos, Perú. Test Engineering $\mathcal{E}$ Management, 83, 9236 - 9242. http://www.testmagzine.biz/index. $\mathrm{php} /$ testmagzine/article/view/5279

Hoyos, A., Ramirez, A., Fernandez, V., \& Sanchez, N. E. (2016). Lenteja de agua (Lemna minor) para el tratamiento de las aguas residuales que provienen del lavado de la fibra de fique (Furcraea bedinghausii). Ingenieria y Competividad, 18(2), 25-34. https://doi.org/10.25100/iyc.v18i2.2151

Jojoa, G., Rodríguez, H., \& Gardona, S. (2015). Tratamiento de aguas residuales textiles a partir de métodos biológicos. Revista CINTEX, 20(1), 11-34. https://www.academia.edu/38191080/ Tratamiento_de_aguas_residuales_textiles_a_partir_de_m\%C3\%A9todos_biol\% C $3 \%$ B3gicos 
Li, H., Mo, F., Li, Y., Wang, M., Li, Z., Hu, H., Deng, W., \& Zhang, R. (2020). Effects of silver (I) toxicity on microstructure, biochemical activities, and genic material of Lemna minor L. with special reference to application of bioindicator. Environmental Science \& Pollution Research, 27(18), 22735-22748. https://doi.org/10.1007/s11356-020-08844-8

Rodriguez, C., Esenarro, D., Gcorimanya, P., Flores, F., Aylas, C., \& Lagos, J. (2020). Proposal for a sustainable infrastructure design (ecolodge) in the Quichas Town, Perú. Test Engineering and Management, 83, 9250-9256. http://www.testmagzine.biz/index.php/testmagzine/ article/view/5281

Sarango, J. A., Sánchez, S., \& Landívar, J. (2016). Educación ambiental. ¿Por qué la Historia?. Revista Universidad y Sociedad, 8(3), 184-187. http://scielo.sld.cu/scielo.php?script=sci_ abstract\&pid=S2218-36202016000300025\&lng=es\&nrm=iso

Sun, Y., Gao, P., Ding, N., Zou, X., Ghen, Y., Li, T., Guiting, W., Xu, X., Ghen, T., Ruan, H. (2020). Feasible Green Strategy for the Quantitative Bioaccumulation of Heavy Metals by Lemna minor: Application of the Self-Thinning Law. Bulletin of Environmental Contamination EF Toxicology, 104(2), 282-287. https://doi.org/10.1007/s00128-019-02772-1

Walsh, É., Paolacci, S., Burnell, G., Jansen, M. A. K. (2020). The importance of the calcium-tomagnesium ratio for phytoremediation of dairy industry wastewater using the aquatic plant Lemna minor L. International Fournal of Phytoremediation, 22(7), 694-702. https://doi.org/10.1080/152265 14.2019 .1707478 


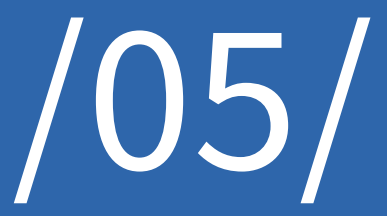




\title{
ALTERNATE FORMULA FOR CALCULATING THE DARCY COEFFICIENT IN TURBULENT FLOW IN PIPES
}

\author{
Freddy Lizardo Kaseng Solis \\ National University Federico Villarreal, (Perú). \\ E-mail: fkaseng@unfv.edu.pe ORCID: https:// orcid.org/0000-0002-2878-9053
}

Remo Bayona Antúnez

National University Federico Villarreal, (Perú).

E-mail: remobayona@yahoo.com ORCID: https:// orcid.org/0000-0001-8655-1193

Giro Rodriguez Rodriguez

National University Mayor de San Marcos, (Perú).

E-mail: crodriguezro@unmsm.edu.pe ORCID: https:// orcid.org/0000-0003-2112-1349

\section{Citación sugerida:}

Kaseng, F.L., Bayona, R., y Rodriguez, C. (2020). Alternate formula for calculating the Darcy Coefficient in turbulent flow in pipes. 3C Tecnologia. Glosas de innovación aplicadas a la pyme, 9(3), 99-109. https://doi.org/10.17993/3ctecno/2020. v9n3e35.99-109 


\section{ABSTRACT}

The purpose of this research was to determine an alternative formula for calculating the Darcy coefficient in turbulent flow in pipes. The proposed alternate formula is an explicit formula that should be used to replace the Colebrook-White formula for calculating the Darcy coefficient in turbulent flow in pipes since it has higher precision than the explicit formulas that are currently in use. In this investigation, the alternate formula was compared with two explicit formulas commonly used in pipe design, the Swamee-Jain and Pavlov formulas. To determining which formula is better, all of them were compared with the Colebrook-White formula. For this, the average percentage and maximum percentage errors of the Darcy coefficient values calculated with each of the explicit formulas were determined, with the values obtained with the Colebrook - White formula. It was determined that the maximum errors in the calculation of the Darcy coefficient concerning the Colebrook-White formula were: 3,104\% for the Swamee-Jain formula, 7,973\% for the Pavlov formula and 2,740\% for the alternate formula.

\section{KEYWORDS}

Pipes, Turbulent Flow, Darcy Coefficient, Colebrook-White Formula, Swamee-Jain Formula, Pavlov Formula, Alternate Formula. 


\section{INTRODUCTION}

An important part of the design of simple or complex hydraulic systems is the calculation of pressure pipes. As in all calculations, the designer seeks precision and simplicity, which are opposed, since generally, the simplicity carries with it the loss of accuracy. That loss of precision must be as little as possible for the simplification to make sense since a significant loss of precision would make the proposed simplification inappropriate.

A well-known formula for calculating the Darcy coefficient for turbulent flow in pipes is the ColebrookWhite formula. This formula has been used to prepare graphs for determining the Darcy coefficient, as is the case of the Moody diagram. However, the Colebrook-White formula has the drawback of being an implicit formula, which has to be solved by successive approximations, which is inconvenient for the calculation.

There are many explicit formulas to solve this problem that have been proposed that try to approximate the results obtained with the Colebrook-White formula. Anaya et al. (2014) indicate that Pavlov's formula is the most recommended to replace the Colebrook-White implicit formula. According to Mott (2006), the Swamee-Jain formula produces values for the Darcy coefficient, which are within $\pm 1.0 \%$ of the value of those corresponding to the Colebrook-White equation, within the range of relative roughness between 0.001 and 1x10-6 and for Reynolds numbers ranging from 5x103 to 1x108.

In the present research, it was demonstrated that an alternative formula, proposed by the author, has higher precision than the formulas that are mentioned and that are currently used, classification algorithms could be used as Huapaya et al. (2020), and Levy et al. (2020).

\section{MATERIAL AND METHODS}

The research design was quasi-experimental; because variables were manipulated to obtain Darcy coefficients by different formulas. 
According to Spiegel and Stephens (2009), the sample size as for an infinite or unknown population is:

$$
n=\frac{Z_{\alpha}^{2} p q}{i^{2}}
$$

Where:

$\mathrm{n}$ : sample size

Za: value corresponding to the Gaussian distribution

$\mathrm{p}$ : expected prevalence of the parameter to be evaluated, if unknown $(\mathrm{p}=0.5)$, which increases the sample size

$i$ : error

Sampling was carried out at the discretion of the researcher, proposing the values of Reynolds numbers and relative roughness indicated above. The samples were obtained by calculating through the respective formula (Colebrook - White, Swamee Jain, Pavlov, and alternate formula) the Darcy coefficients corresponding to predefined values of Reynolds numbers and relative roughness. The Reynolds number and relative roughness values used to obtain the sample were evenly distributed within the limits for which the Colebrook-White formula is valid, from 4000 to 108 for the Reynolds number and from 0.05 to 10-8 for the relative roughness.

The Darcy coefficient depends on the Reynolds number Re and the relative roughness $\varepsilon$ r.

Values of Reynolds numbers and relative roughnesses within the ranges of application of the ColebrookWhite formula were proposed, and the respective Darcy coefficients were determined with the ColebrookWhite, Swamee-Jain, Pavlov formulas and the alternate formula.

$$
\frac{1}{\sqrt{f}}=-2 \log \left(\frac{\varepsilon_{r}}{3.7}+\frac{2.51}{\operatorname{Re} \sqrt{f}}\right) \quad \text { Colebrook - White }
$$




$$
\begin{array}{ll}
f=\frac{0.25}{\left[\log \left(\frac{\varepsilon_{r}}{3.7}+\frac{5.74}{R e^{0.9}}\right)\right]^{2}} & \text { Swamee - Jain } \\
f=\frac{0.25}{\left[\log \left(\frac{\varepsilon_{r}}{3.7}+\frac{6.81}{R e^{0.9}}\right)\right]^{2}} & \text { Pavlov } \\
f=\frac{0.25}{\log \left(\frac{1}{3.7}\left\{\frac{\epsilon}{D}\right\}+\frac{6.81}{R e^{0.9}}\right)^{2}} \quad \text { Alternate formula }
\end{array}
$$

It was worked with a sample of 70007 Darcy coefficient values for each of the formulas used, comparing each of the explicit formulas (Swamee - Jain, Pavlov, and the alternate formula) with the implicit Colebrook - White formula.

For each of the explicit formulas, the mean percentage error em and the maximum percentage error Emax were obtained for the Colebrook White formula. Then these errors were compared with each other to determine which of the formulas is the most appropriate for the calculation of the Darcy coefficient in pipes with turbulent flow.

The calculations were carried out on an Excel spreadsheet, resulting in Table 1. 
Table 1. Calculation of the percentage errors of the explicit formulas to the Colebrook - White formula.

\begin{tabular}{|c|c|c|c|c|c|c|c|c|c|c|c|c|c|c|c|}
\hline \multirow[b]{2}{*}{$\mathbf{N}^{\circ}$} & \multirow[b]{2}{*}{$\operatorname{Re}$} & \multirow[b]{2}{*}{$\varepsilon_{r}$} & \multirow{2}{*}{$\begin{array}{c}\text { Colebrook } \\
\mathrm{f}\end{array}$} & \multicolumn{4}{|c|}{ Swamee - Jain } & \multicolumn{4}{|c|}{ Pavlov } & \multicolumn{4}{|c|}{ Alternate formula } \\
\hline & & & & $f_{1}$ & $e_{1}$ & $\left|e_{1}\right|$ & $e_{m}$ & $f_{2}$ & $e_{2}$ & $\left|e_{2}\right|$ & $e_{m}$ & $f_{3}$ & $e_{3}$ & $\left|e_{3}\right|$ & $e_{m}$ \\
\hline 1 & 4000 & 0,05 & 0,07699 & 0,0794 & 3,104 & 3,104 & 0,551 & 0,08079 & 4,936 & 4,936 & 1,683 & 0,07885 & 2,416 & 2,416 & 0,236 \\
\hline 2 & 4000 & $1,00 \mathrm{E}-03$ & 0,04091 & 0,0417 & 1,931 & 1,931 & $\mathbf{e}_{\max }$ & 0,04415 & 5,875 & 7,920 & $\mathbf{e}_{\max }$ & 0,04073 & $-0,440$ & 0,440 & $e_{\max }$ \\
\hline 3 & 4000 & $1,00 \mathrm{E}-04$ & 0,04001 & 0,0407 & 1,650 & 1,650 & 3,104 & 0,0432 & 6,221 & 7,973 & 7,973 & 0,03967 & $-0,850$ & 0,850 & 2,416 \\
\hline 4 & 4000 & $1,00 \mathrm{E}-05$ & 0,03992 & 0,0406 & 1,603 & 1,603 & & 0,0431 & 6,262 & 7,966 & & 0,03956 & $-0,902$ & 0,902 & \\
\hline 5 & 4000 & $1,00 \mathrm{E}-06$ & 0,03991 & 0,0406 & 1,604 & 1,604 & & 0,04309 & 6,264 & 7,968 & & 0,03955 & $-0,902$ & 0,902 & \\
\hline 6 & 4000 & $1,00 \mathrm{E}-07$ & 0,03991 & 0,0406 & 1,604 & 1,604 & & 0,04309 & 6,264 & 7,968 & & 0,03955 & $-0,902$ & 0,902 & \\
\hline 7 & 4000 & $1,00 \mathrm{E}-08$ & 0,03991 & 0,0406 & 1,604 & 1,604 & & 0,04309 & 6,264 & 7,968 & & 0,03955 & $-0,902$ & 0,902 & \\
\hline 8 & 10000 & 0,05 & 0,07178 & 0,072 & 0,306 & 0,306 & & 0,07208 & 0,111 & 0,418 & & 0,07197 & 0,265 & 0,265 & \\
\hline 9 & 10000 & $1,00 \mathrm{E}-03$ & 0,02217 & 0,0223 & 0,767 & 0,767 & & 0,02277 & 1,926 & 2,706 & & 0,02218 & 0,045 & 0,045 & \\
\hline 10 & 10000 & $1,00 \mathrm{E}-04$ & 0,01851 & 0,0185 & $-0,324$ & 0,324 & & 0,01913 & 3,686 & 3,350 & & 0,01818 & $-1,783$ & 1,783 & \\
\hline 11 & 10000 & $1,00 \mathrm{E}-05$ & 0,01804 & 0,0179 & $-0,665$ & 0,665 & & 0,01865 & 4,074 & 3,381 & & 0,01763 & $-2,273$ & 2,273 & \\
\hline 12 & 10000 & $1,00 \mathrm{E}-06$ & 0,018 & 0,0179 & $-0,722$ & 0,722 & & 0,0186 & 4,085 & 3,333 & & 0,01757 & $-2,389$ & 2,389 & \\
\hline 13 & 10000 & $1,00 \mathrm{E}-07$ & 0,01799 & 0,0179 & $-0,723$ & 0,723 & & 0,01859 & 4,087 & 3,335 & & 0,01757 & $-2,335$ & 2,335 & \\
\hline 14 & 10000 & $1,00 \mathrm{E}-08$ & 0,01799 & 0,0179 & $-0,723$ & 0,723 & & 0,01859 & 4,087 & 3,335 & & 0,01757 & $-2,335$ & 2,335 & \\
\hline 15 & 20000 & 0,05 & 0,07167 & 0,0718 & 0,167 & 0,167 & & 0,07183 & 0,056 & 0,223 & & 0,07177 & 0,140 & 0,140 & \\
\hline
\end{tabular}

Source: authors' own elaboration.

Table 1 shows the following percentage errors:

- $\quad$ el (percentage error of the Swamee - Jain formula to the Colebrook - White formula)

- $\quad$ 2 (percentage error of Pavlov's formula to Colebrook-White's formula)

- $\quad$ e3 (percentage error of the alternate formula to the Colebrook - White formula)

These errors are determined using the following formulas:

$$
\begin{aligned}
& e_{3}=\frac{f_{3}-f}{f} \cdot 100 \% \\
& e_{2}=\frac{f_{2}-f}{f} \cdot 100 \% \\
& e_{1}=\frac{f_{1}-f}{f} \cdot 100 \%
\end{aligned}
$$


Where:

$\mathrm{f}$ is the value of the Darcy coefficient calculated with the Colebrook - White formula.

$\mathrm{fl}$ is the value of the Darcy coefficient calculated with the Swamee - Jain formula.

$\mathrm{f} 2$ is the value of the Darcy coefficient calculated with the Pavlov formula.

$\mathrm{f} 3$ is the value of the Darcy coefficient calculated with the alternative formula.

\section{RESULTS}

The testing of the hypothesis was performed by comparing the mean and maximum percentage errors, obtained from the comparison between the Darcy coefficients calculated with each of the explicit formulas, and the Darcy coefficients obtained by the Colebrook-White formula.

- emed 1 and emax 1 the mean percentage and maximum percentage errors obtained when calculating the Darcy coefficients with the Swamee-Jain formula, compared to those obtained using the Colebrook-White formula.

- emed2 and emax2 the mean percentage and maximum percentage errors obtained when calculating Darcy coefficients with the Pavlov formula, compared to those obtained using the Colebrook White formula.

The summary of the errors is shown in Table 2:

Table 2. Percentage errors of explicit formulas.

\begin{tabular}{|c|c|c|c|}
\hline & Swamee - Jain & Pavlov & Alternate \\
\hline $\mathbf{e}_{\mathbf{m}}$ & 0,551 & 1,683 & 0,236 \\
\hline $\mathbf{e}_{\max }$ & 3,104 & 7,973 & 2,416 \\
\hline
\end{tabular}

Source: authors' own elaboration. 
As can be seen, for the sample used, the alternate formula presents a mean percentage error of $0.236 \%$ and a maximum percentage error of $2.416 \%$ on the Colebrook-White formula. Both values are significantly smaller than the errors in the Swamee - Jain, and Pavlov formulas.

\section{DISCUSSION}

The results do not agree with the results obtained by Anaya et al. (2014), who propose Pavlov's formula for calculating the Darcy coefficient. The use of a single relative roughness value of 0.001 in that investigation may have led to less than exact conclusions.

The statement of Mott (2006) is confirmed in that the Swamee-Jain formula is a good alternative for calculating the Darcy coefficient for turbulent flow in pipes.

The alternate formula outperforms the other formulas. It has an average error equal to $42.7 \%$ of the average error of the Swamee - Jain formula and equivalent to $14 \%$ of the error of the Pavlov formula. As for the maximum error, this is $77.8 \%$ of the maximum error of the Swamee-Jain formula and $30.3 \%$ of the maximum error of the Pavlov formula.

It is concluded that the alternative formula is the best option for calculating the Darcy coefficient for turbulent flow in pipes.

\section{CONCLUSION}

The alternate formula outperforms the other formulas. It has an average error equal to $42.7 \%$ of the average error of the Swamee - Jain formula and equivalent to $14 \%$ of the error of the Pavlov formula. As for the maximum error, this is $77.8 \%$ of the maximum error of the Swamee-Jain formula and $30.3 \%$ of the maximum error of the Pavlov formula. 
It is concluded that the alternative formula is the best option for calculating the Darcy coefficient for turbulent flow in pipes.

\section{REFERENCES}

Anaya, A. I., Gauich, G. I., Funabazama, O., \& Gracia, V. A. (2014). Evaluación de ecuaciones del factor de fricción explícito en tuberías. Educación Química, 128-134.

Camaraza, Y., \& García, O. F. (2008). Ecuación explícita para el cálculo de factores de fricción en la zona de transición del flujo turbulento. Universidad de Matanzas "Camilo Cienfuegos".

Escuela Universitaria de Ingeniería Técnica Agrícola de Giudad Real. (s.f.). Expresiones del factor de fricción. Ciudad Real. https://previa.uclm.es/area/ing_rural/Hidraulica/Temas/Tema7.pdf

Fernández, B. (1999). Introducción a la Mecánica de Fluidos. Alfaomega grupo editor SA.

Giles, R., Evett, J., \& Liu, C. (1980). Mecánica de los Fluidos e Hidráulica. Mc Graw Hill.

Guerrero, J. O. (1995). Ecuación modificada de Colebrook - White. Ingeniería Hidráulica en México, 43-48.

Huapaya, H. D., Rodriguez, G., \& Esenarro, D. (2020). Comparative analysis of supervised machine learning algorithms for heart disease detection. 3C Tecnología. Glosas De Innovación Aplicadas a La Pyme, 233-247. https://ojs.3ciencias.com/index.php/3c-tecnologia/article/view/1003

Levy, J., Pandey, B., Chowdhry, B., \& Rodriguez, C. (2020). Prologue: Recent trends in computer science and engineering (RTCSE). 3C Tecnología. Glosas De Innovación Aplicadas a La Pyme, 19-25. https://ojs.3ciencias.com/index.php/3c-tecnologia/article/view/991

Mott, R. (2006). Mecánica de Fluidos Aplicada. Prentice Hall Hispanoamericana SA.

Munson, B., Young, D., \& Okiishi, T. (1999). Fundamentos de Mecánica de Fluidos. Editorial Limusa SA. 
Perez, D., \& Echeverría, J. (2002). Evolución histórica de las fórmulas para expresar las pérdidas de carga en tuberías. Ingeniería Hidráulica y Ambiental.

Saldarriaga, J. (1998). Hidráulica de tuberías. Mc Graw Hill Interamericana SA.

Shames, I. (1995). Mecánica de Fluidos. Mc Graw Hill.

Soto, B., Vega, H., Guzmán, Y., Rodriguez, G., \& Quinto, D. (2020). Classification algorithm based on machine learning to optimize athletes talent detection. Test Engineering, 83, 13464-13471.

Universidad de Sevilla. (s.f.). Open Course Ware Universidad de Sevilla. http://ocwus.us.es/ingenieriaagroforestal/hidraulica-y-riegos/temario/Tema\%202.Conducciones\%20forzadas/tutorial_03. htm 


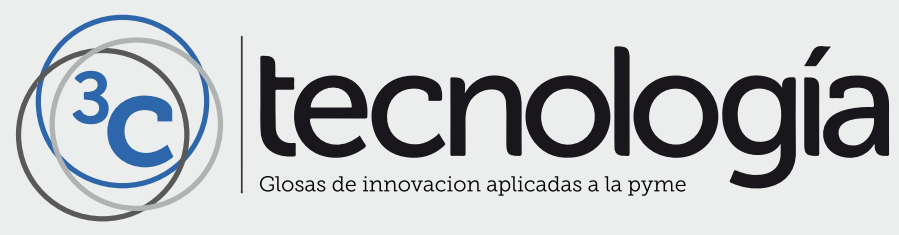

